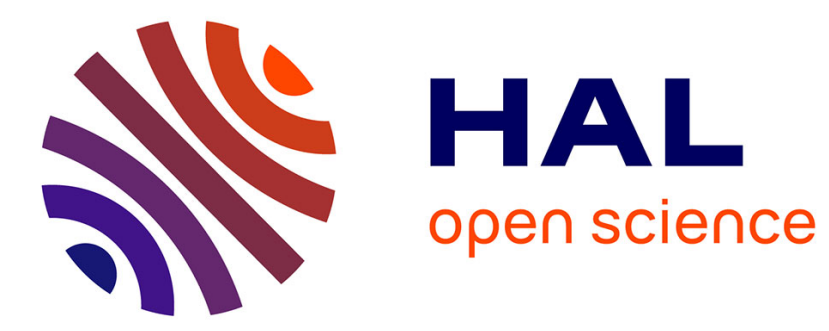

\title{
Proof nets and semi-star-autonomous categories
}

Willem Heijltjes, Lutz Strassburger

\section{To cite this version:}

Willem Heijltjes, Lutz Strassburger. Proof nets and semi-star-autonomous categories. Mathematical Structures in Computer Science, 2014, 26 (5), pp.1-40. 10.1017/S0960129514000395 . hal-01092253

\section{HAL Id: hal-01092253 https://hal.inria.fr/hal-01092253}

Submitted on 8 Dec 2014

HAL is a multi-disciplinary open access archive for the deposit and dissemination of scientific research documents, whether they are published or not. The documents may come from teaching and research institutions in France or abroad, or from public or private research centers.
L'archive ouverte pluridisciplinaire HAL, est destinée au dépôt et à la diffusion de documents scientifiques de niveau recherche, publiés ou non, émanant des établissements d'enseignement et de recherche français ou étrangers, des laboratoires publics ou privés. 


\title{
Proof nets and semi- $\star$-autonomous categories
}

\author{
Willem Heijltjes ${ }^{1}$ and Lutz Straßburger ${ }^{2}$ \\ ${ }^{1}$ Department of Computer Science, University of Bath \\ Claverton Down, Bath BA2 7AY, United Kingdom. \\ w.b.heijltjes@bath.ac.uk \\ ${ }^{2}$ Laboratoire d'Informatique, École Polytechnique \\ Route de Saclay, 91128 Palaiseau, France. \\ lutz@lix.polytechnique.fr
}

Received 26 February 2013, Revised 9 May 2014

In this paper it is proved that Girard's proof nets for multiplicative linear logic characterise free semi- $\star$-autonomous-categories.

\section{Introduction}

The strong geometric intuition to monoidal categories and their relatives means that morphisms can be effectively pictured as graphical objects, such as Kelly-MacLane graphs (Kelly and Mac Lane, 1971), wire diagrams (Joyal and Street, 1991) — see (Selinger, 2011) for an overview, or varieties of linear logic proof nets (Blute, 1993; Blute et al., 1996). In some cases, for example for sum-product categories (Heijltjes, 2011), graphs exist that are canonical: that allow the direct, syntactic construction of the free category.

In this regard, an interesting case is multiplicative linear logic (MLL). Girard's proof nets (Girard, 1987) are canonical for $\mathrm{MLL}^{-}$, multiplicative linear logic without units. However, in star-autonomous categories (Barr, 1991), the semantics of MLL, the units play a central role: as the dualising object, and its dual. The two cases, with and without units, are mathematically distinct, and raise the following two questions: what notion of proof net is canonical for star-autonomous categories, and what notion of category is described by $\mathrm{MLL}^{-}$proof nets?

In one direction, proof nets have been extended with units to capture free star-autonomous categories, in a series of developments (Blute et al., 1996; Lamarche and Straßburger, 2006; Hughes, 2012). These nets are however not canonical, resulting in an equivalence over proof nets imposed by the categorical laws. Although in the formulation by Hughes this equivalence is reduced to a simple rewiring relation, a very recent result is that the associated decision problem - the word problem for star-autonomous categories - is actually PSPACE-complete (Heijltjes and Houston, 2014).

The other direction, pursued further in this paper, concerns the question of what a star-autonomous category without units should be, corresponding to $\mathrm{MLL}^{-}$proof nets. Here, the challenge is to account for proof nets with a single conclusion. Such nets are traditionally modelled by morphisms out of the unit to the tensor, which is unavailable. 
Moreover, the presence of single-conclusion nets cannot be ignored: as in the example below, they may occur as subnets of multi-conclusion nets, which are to be interpreted as categorical morphisms.

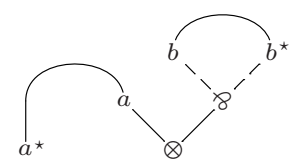

In the recent past two approaches to this problem have been put forward. One, first explored by Lamarche and Straßburger (Lamarche and Straßburger, 2005), proposes to replace the monoidal unit $I$ in a star-autonomous category $\mathcal{C}$ by a 'virtual unit', a functor $\mathbb{I}: \mathcal{C} \rightarrow$ SET that takes the rôle of $\operatorname{hom}(I,-)$. This is also the direction taken in the thesis of Houston (Houston, 2008), who defines the notion of semi-star-autonomous categories from the more abstract perspective of monoidal bicategories and promonoidal categories (on which more below). The present paper aims to complete this line of research, reviewing and relating the necessary axioms, and proving that $\mathrm{MLL}^{-}$proof nets characterise free semi-star-autonomous categories.

A second approach, 'proof net categories' by Došen and Petrić (Došen and Petrić, 2005), is to omit the unit from linearly distributive categories (Cockett and Seely, 1997) and add a natural transformation $A \Rightarrow A \otimes\left(B^{\star} \diamond B\right)$. The corresponding operation on a proof net is to endow it with a trivial single-conclusion subnet, as in the above example; non-trivial such subnets are then constructed using associativity, symmetry, and the functoriality of the tensor. While the approach has the advantage of presenting a regular categorical structure, without the need for a virtual unit functor, it also has certain drawbacks. Firstly, definitions of linearly distributive categories involve many coherence axioms, and to recover the traditional structure associated with star-autonomous categories, in particular the defining adjunction connecting the tensor and the par, is non-trivial. Secondly, proof net categories do not actually incorporate single-conclusion proof nets as individual nets, but only as subnets, which moreover are constructed in a roundabout fashion. Without reflecting major problems, these points do mean that important intuitions about proof nets are lost.

\section{Promonoidal categories}

The ideas behind the virtual unit functor $\mathbb{I}: \mathcal{C} \rightarrow$ SET first appeared in the work of Day (Day, 1970) on promonoidal categories (there called premonoidal categories). These are a generalisation of monoidal categories where the tensor and unit are given by profunctors

$$
P: \mathcal{C} \times \mathcal{C} \rightarrow \mathcal{C} \quad J: 1 \rightarrow \mathcal{C} .
$$

Monoidal categories are then the special case where both profunctors are represented by actual functors. In this light, semi-star-autonomous categories are a form of promonoidal category where the tensor is representable, but the unit need not be: the unit profunctor is exactly the virtual unit functor $\mathbb{I}$. Houston in his thesis (Houston, 2008) explores this path towards semi-star-autonomous categories in still greater generality, first developing the 
theory of monoidal bicategories, in which promonoidal categories occur as a construction called a pseudomonoid.

The abstraction offered by promonoidal categories means that reasoning about the virtual unit $\mathbb{I}$ proceeds along similar lines to standard reasoning in monoidal categoriesthis in contrast to reasoning with $\mathbb{I}$ within the internal language of semi-star-autonomous categories, which may often be convoluted and counterintuitive. In particular, this concerns the proof that the symmetry isomorphism acts as desired on nets consisting of a tensor of two single-conclusion nets, as illustrated schematically below.

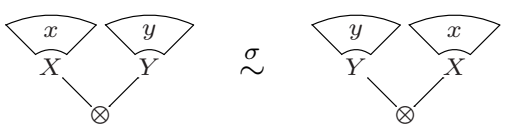

While the promonoidal proof of this equation is a direct rendition of Kelly's (Kelly, 1964) proof that the left and right unit isomorphisms $\lambda_{I}, \rho_{I}: I \otimes I \rightarrow I$ commute, its internal translation into semi-star-autonomous categories produces a proof that is far from obvious - as witnessed by the fact that this equation was taken as an axiom in (Lamarche and Straßburger, 2005).

What this illustrates is that the gap between promonoidal categories and proof nets is considerable, while the abstract approach of Houston (Houston, 2008) leaves many details implicit. In bridging this gap, this paper assumes the modest task of giving a direct proof that proof nets characterise free semi-star-autonomous categories. While similar proofs have been carried out for proof nets with units, some are rather indirect (Blute et al., 1996; Hughes, 2012), and others rely heavily on the presence of the unit in star-autonomous categories (Lamarche and Straßburger, 2006). Here, the aim is to present a proof that is direct and clear, and on the way explicate and concretise the results of (Houston, 2008).

\section{Proof nets}

The grammar below describes $\mathrm{MLL}^{-}$-formulae over a set of atoms $a, b, \ldots \in \mathcal{A}$.

$$
A:=a\left|a^{\star}\right| A \otimes A \mid A \& A
$$

Negation $A^{\star}$ for general formulae $A$ is via DeMorgan (see also below). Sequents will be used in one-sided form $\Gamma$ and two-sided form $\Gamma \vdash \Delta$, where $\Gamma$ and $\Delta$ are multisets of formulae.

Let an annotated formula $A_{V}$ be a formula $A$ annotated by a set of vertices $V$, according to the following grammar (where $\uplus$ indicates union of disjoint sets).

$$
A_{V}:=\underbrace{a_{v} \mid a_{v}^{\star}}_{V=\{v\}} \mid \underbrace{B_{U} \otimes_{v} C_{W} \mid B_{U} 8_{v} C_{W}}_{V=U \uplus\{v\} \uplus W}
$$

Similarly, let an annotated sequent $\Gamma_{V}$ or $\Gamma_{V} \vdash \Delta_{W}$ be one whose formulae are annotated distinctly. The purpose of annotating formulae and sequents in this way is threefold. Firstly, by naming its nodes a formula tree is made into a graphical object, to which the axiom links of proof nets may be connected directly. Secondly, the annotation removes the 
$\frac{\bar{f}}{\{\langle v, w\rangle\}:\left[a_{v}, a_{w}^{\star}\right]} \mathbf{A X} \quad \frac{\mathcal{L}:\left[\Gamma_{X}, A_{V}, B_{W}\right]}{\mathcal{L}:\left[\Gamma_{X}, A_{V} \gamma_{u} B_{W}\right]} \ngtr \mathbf{R} \quad \frac{\mathcal{L}:\left[\Gamma_{X}, A_{V}\right] \quad \mathcal{K}:\left[\Delta_{Y}, B_{W}\right]}{\mathcal{L} \cup \mathcal{K}:\left[\Gamma_{X}, \Delta_{Y}, A_{V} \otimes_{u} B_{W}\right]} \otimes \mathbf{R}$

Fig. 1. Constructing proof nets by the calculus LM

need to distinguish between subformulae and subformula occurrences, and allows precise and consistent reference to the latter. Thirdly, annotation allows easy formulation of coherence properties in $\mathrm{MLL}^{-}$as a category, addressed in Section 7 .

In an annotated formula, a tensor-vertex $v$ in $A_{V}$ is one annotating a tensor $\left(\otimes_{v}\right)$, a par-vertex annotates a par $\left(\gamma_{v}\right)$, and an atomic vertex annotates an atom $\left(a_{v}\right)$ or negated atom $\left(a_{v}^{\star}\right)$. The root vertex of an annotated (sub)formula is that of its primary connective; the formula of a vertex $v$ is the formula occurrence of which $v$ is the root; and the children of a par-vertex or tensor-vertex are the root vertices of its subformulae. The root vertices of a sequent are also called its ports. In an annotated formula or sequent, two vertices are called dual if their formulae are dual. In dualising an annotated formula $A_{V}$ to $A_{V}^{\star}$, the annotation is preserved:

$$
a_{v}^{\star \star}=a_{v} \quad\left(A_{V} \otimes_{u} B_{W}\right)^{\star}=A_{V}^{\star} 8_{u} B_{W}^{\star} \quad\left(A_{V} \gamma_{u} B_{W}\right)^{\star}=A_{V}^{\star} \otimes_{u} B_{W}^{\star}
$$

Similarly, an anotated sequent $\Gamma_{V}$ can be dualised to $\Gamma_{V}^{\star}$.

Definition 2.1. A pre-proof net (or prenet) $\mathcal{L}:\left[\Gamma_{V}\right]$ consists of an annotated sequent $\Gamma_{V}$ and a linking $\mathcal{L}$, which is a partitioning of the atomic vertices in $V$ into dual (unordered) pairs.

A switching graph for a prenet $\mathcal{L}:\left[\Gamma_{V}\right]$ is an undirected graph $(V, E \cup \mathcal{L})$ where for every tensor-vertex $v$ in $\Gamma_{V}$ the set $E$ contains all edges $\langle v, w\rangle$ connecting $v$ to its children $w$, and for every par-vertex $v$ the set $E$ contains exactly one edge $\langle v, w\rangle$ connecting $v$ to one of its children $w$. Note that a proof net with $n$ par-vertices has $2^{n}$ switching graphs.

Definition 2.2 ((Danos and Regnier, 1989)). A proof net is a prenet for which every switching graph is acyclic and connected.

In Figure 1 the standard sequent calculus for $\mathrm{MLL}^{-}$is adapted to make the construction of proof nets by sequent proofs explicit. A derivation from a number of premisses to one conclusion, consisting of several inference rules, will be abbreviated by a double horizontal bar; a proof is a derivation from zero premisses. The net constructed by a proof $\Pi$ will be denoted $\llbracket \Pi \rrbracket$.

Proposition 2.3 ((Danos and Regnier, 1989)). Proof nets are precisely the prenets constructed by the sequent calculus LM in Figure 1 .

Describing the category of proof nets first requires to interpret nets as morphisms.

Definition 2.4. A two-sided proof net $\mathcal{L}:\left[\Gamma_{V} \vdash \Delta_{W}\right]$ consists of a two-sided sequent $\Gamma_{V} \vdash \Delta_{W}$ plus a linking $\mathcal{L}$, such that $\mathcal{L}:\left[\Gamma_{V}^{\star}, \Delta_{W}\right]$ is a (one-sided) proof net.

A proof net of the form $\mathcal{L}:\left[A_{V} \vdash B_{W}\right]$ may then be interpreted as a morphism $A \rightarrow B$. 


$$
\begin{aligned}
& \frac{\overline{\overline{\Gamma, a}} \quad \overline{a^{\star}, a}}{\Gamma, a} \text { AX } \\
& \rightsquigarrow \quad \overline{\overline{\Gamma, a}}
\end{aligned}
$$

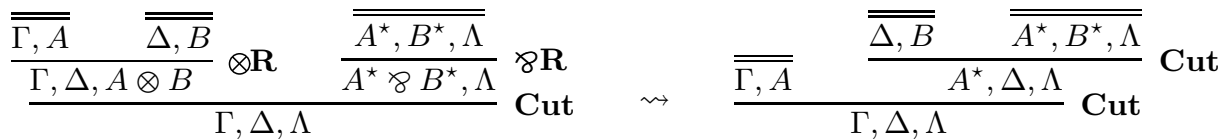

Fig. 2. Cut reduction steps in LM

For a proof net $\mathcal{L}:\left[\Gamma_{U}\right]$, over a sequent $\Gamma$ of a more general form, it is not always possible to find a single, unambiguous, corresponding morphism. This is because $\Gamma$ does not unambiguously denote a source and target object in the category, but instead a range of possible such pairs of objects. Correspondingly, a proof net $\mathcal{L}:\left[\Gamma_{U}\right]$ may be interpreted by a class of morphisms, containing precisely the following: the interpretation of each proof net $\mathcal{L}:\left[A_{V} \vdash B_{W}\right]$ and $\mathcal{L}:\left[B_{W}^{\star} \vdash A_{V}^{\star}\right]$ such that $\mathcal{L}:\left[A_{V}^{\star}, B_{W}\right]$ is derivable from $\mathcal{L}:\left[\Gamma_{U}\right]$ by just $\varnothing \mathbf{R}$-inferences.

Next, the primary categorical structure on proof nets, composition and identities, will be treated. The composition of two proof nets will be defined directly via pathcomposition, following Hughes (Hughes, 2012). In contrast, the approach of Girard (Girard, 1987) introduces a cut into the proof net structure, which is then eliminated via local rewriting operations similar to those on proofs.

Definition 2.5. The composition of two proof nets is given by the rule

$$
\frac{\mathcal{L}:\left[\Gamma_{U}, A_{V}\right] \quad \mathcal{K}:\left[A_{V}^{\star}, \Delta_{W}\right]}{\mathcal{L} ; \mathcal{K}:\left[\Gamma_{U}, \Delta_{W}\right]} \mathbf{C u t}
$$

where $U \cap W=\emptyset$ and where $\mathcal{L} ; \mathcal{K}$ contains a link $\langle u, w\rangle$ precisely when there is a path of links $\left\langle u, v_{1}\right\rangle,\left\langle v_{1}, v_{2}\right\rangle, \ldots,\left\langle v_{n}, w\right\rangle$ (alternately) from $\mathcal{L}$ and from $\mathcal{K}$.

In the above definition the formulae $A_{V}^{\star}$ and $A_{V}$ are the cut-formulae. The characterisation of $\mathcal{L} ; \mathcal{K}$ as path-composition in $\mathcal{L} \cup \mathcal{K}$ relies on the fact that $\mathcal{L}$ and $\mathcal{K}$ share only vertices in $V$, and means the composition of two nets (over given cut-formulae) is uniquely defined.

Cut-elimination is defined by the reduction rules displayed in Figure 2 (plus the two symmetric variants obtained by swapping the two subproofs of each). The following proposition is well-known and straightforward to show:

Proposition 2.6 ((Girard, 1987; Hughes, 2012)). The rule Cut is admissible in the calculus LM; moreover, cut-elimination preserves the net constructed by a proof.

Composition of nets is associative, and unitary with respect to a class of identity nets, 


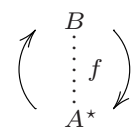<smiles>B1OC[C]CO1</smiles>

$f^{\star}$

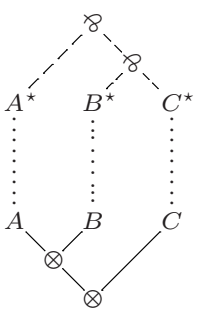

$\alpha_{A, B, C}$

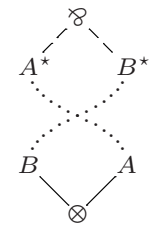<smiles>[Y1][C@@]12[B][C@@H]1O[B]O[B]C2</smiles><smiles>[CH][C@]1(C)[B]OO[B]C1</smiles>

$\eta_{A, B}$

$\epsilon_{B, C}$

Fig. 3. Categorical constructions in proof nets

constructed by the axiom rule and the following composite rule.

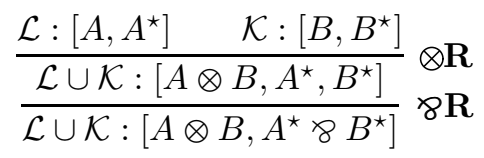

Further structural morphisms in the category of proof nets are illustrated in Figure 3 (note that the dotted arcs represent subnets: $f$ (for $A \vdash B$ ) or $g$ (for $C \vdash D$ ) when indicated, and identity nets otherwise). While omitting the unit, the category of proof nets retains much of the structure of a star-autonomous category: an associative and symmetric tensor-bifunctor, an involutive duality functor $-^{\star}$, and an adjunction between functors $A \otimes-$ and $\left(-\otimes A^{\star}\right)^{\star}$. In the next section a notion of tensor-dual category with just this structure will be defined. However, tensor-dual categories capture only a subclass of proof nets: using just the morphisms in Figure 3, which all have both a source object and a target object, it is impossible to construct a single-conclusion proof net $\mathcal{L}:\left[A_{V}\right]$, nor one with single-conclusion subnets.

Definition 2.7. A prenet $\mathcal{K}:\left[\Delta_{W}\right]$ is a subprenet of a prenet $\mathcal{L}:\left[\Gamma_{V}\right]$ if

- $\Delta_{W}$ consists of subformula occurrences of $\Gamma_{V}$, i.e. $W \subseteq V$ and every vertex $w \in W$ is the root of the same annotated formula in $\Delta_{W}$ and $\Gamma_{V}$; and

- $\mathcal{K}$ is the restriction of $\mathcal{L}$ to $W$.

A subnet is a subprenet that is a proof net.

Definition 2.8. A tensor-dual net (TD-net) is a two-sided proof net with exactly two ports $\mathcal{L}:\left[A_{V} \vdash B_{W}\right]$, such that every subnet has at least two ports. Let $\operatorname{TDNET}(\mathcal{A})$ denote the category of TD-nets over the set of atoms $\mathcal{A}$.

\section{Tensor-dual categories}

In the previous section the class of tensor-dual nets was identified because it has a conventional categorical semantics, in the tensor-dual categories defined below.

Definition 3.1. A tensor-dual category (TD category) $\left(\mathcal{C}, \otimes,{ }^{*}\right)$ is a category $\mathcal{C}$ with a tensor bifunctor $(-\otimes-)$ and a dualising functor $(-)^{\star}$ on $\mathcal{C}$, with the following natural 


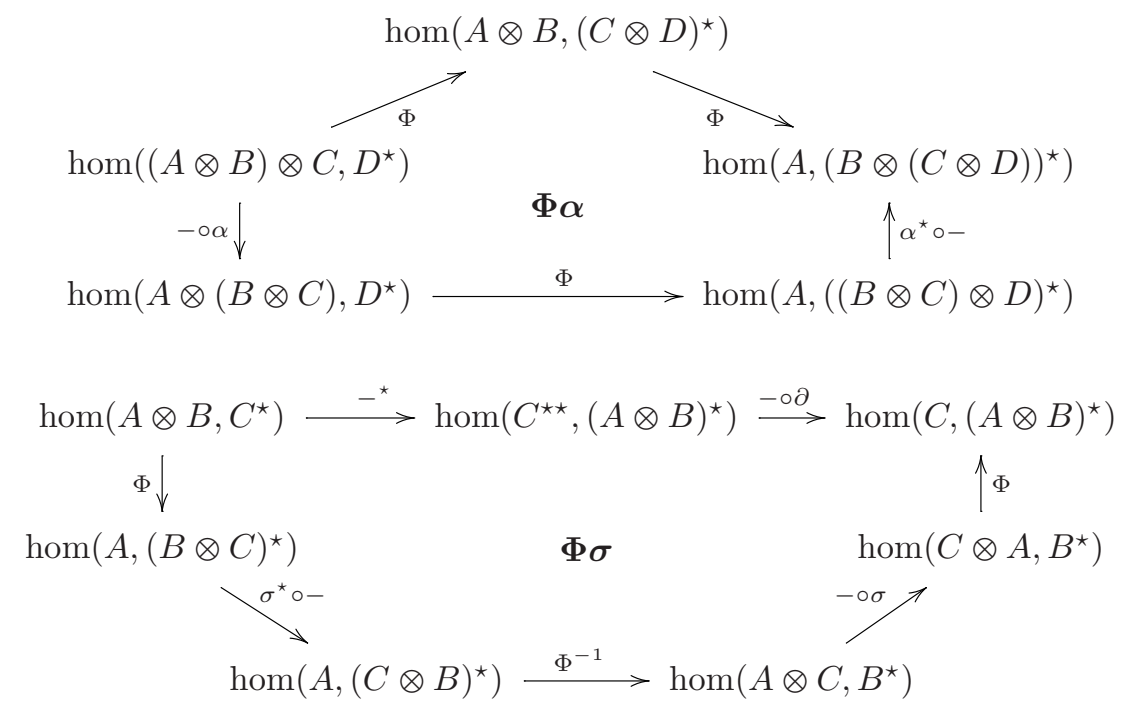

Fig. 4. Coherence axioms for tensor-dual categories

isomorphisms,

$$
\begin{array}{cc}
\alpha: A \otimes(B \otimes C) \cong(A \otimes B) \otimes C & \sigma: A \otimes B \cong B \otimes A \\
\partial: A \cong A^{\star \star} & \Phi: \operatorname{hom}\left(A \otimes B, C^{\star}\right) \cong \operatorname{hom}\left(A,(B \otimes C)^{\star}\right)
\end{array}
$$

satisfying the familiar pentagon and hexagon diagrams for associativity $(\alpha)$ and symmetry $(\sigma)$, plus those in Figure 4 for $\Phi$ and $-^{\star}$. A tensor-dual functor (TD functor) is a functor between TD categories preserving the structure induced by the functors $-\otimes-$ and $-^{\star}$ up to isomorphism.

The illustration in Figure 3 then shows the following.

Proposition 3.2. For any set $\mathcal{A}$ the category $\operatorname{Tdnet}(\mathcal{A})$ is a tD category.

In the category $\operatorname{TDNET}(\mathcal{A})$ the isomorphism $\partial$ is strict, i.e. $\partial=i d$, due to the treatment of negation via the notation $(A \otimes B)^{\star}=A^{\star} \ngtr B^{\star}$-as opposed to maintaining $(-)^{\star}$ as a syntactic primitive, as for example in (Hughes, 2012). Alternative formulations of $\mathrm{MLL}^{-}$ correspond to various kinds of strictness in tensor-dual categories. The isomorphism $\alpha$ is made strict by using an $n$-ary tensor, and requiring tensor and par to alternate in the construction of a formula. Strictness in $\sigma$ is induced by using, instead of an annotated formula $A_{V}$, the graph it generates over the vertices $V$. The isomorphism $\Phi$ could be made strict by using one-sided rather than two-sided sequents. Note that these adaptations already apply to sequents; however, these are used only during the construction of proof nets, not in the resulting nets that are taken to represent categorical maps. Therefore the category $\operatorname{TDnet}(\mathcal{A})$ is strict only in $\partial$, but not in $\alpha, \sigma$, nor $\Phi$. From here on, $\partial$-strictness will be assumed throughout, except when indicated otherwise, and $A \& B$ will be used to abbreviate $\left(A^{\star} \otimes B^{\star}\right)^{\star}$. 
Note that the central adjunction of TD-categories is generated by the following unit and co-unit, and that only in the $\partial$-strict case $\Phi$ itself is the isomorphism of the adjunction.

$$
\begin{aligned}
\eta_{A, B}=\Phi\left(\partial_{A \otimes B}\right) & : \quad A \rightarrow\left(B \otimes(A \otimes B)^{\star}\right)^{\star} \\
\epsilon_{B, C}=\Phi\left(i d_{(B \otimes C)^{\star}}\right): & (B \otimes C)^{\star} \otimes B \rightarrow C^{\star}
\end{aligned}
$$

Tensor-dual nets characterise free $\partial$-strict TD categories. This is formalised as follows, where $i: \mathcal{A} \rightarrow \operatorname{TDNET}(\mathcal{A})$ is the inclusion taking the atoms in $\mathcal{A}$ into $\operatorname{Tdnet}(\mathcal{A})$.

Theorem 3.3. Any functor $F: \mathcal{A} \rightarrow \mathcal{C}$ into a $\partial$-strict TD category $\mathcal{C}$ factors uniquely (up to natural isomorphism) as $F=G \circ i$, where $G$ : $\operatorname{TDNET}(\mathcal{A}) \rightarrow \mathcal{C}$ is a TD functor, as illustrated by the following diagram.

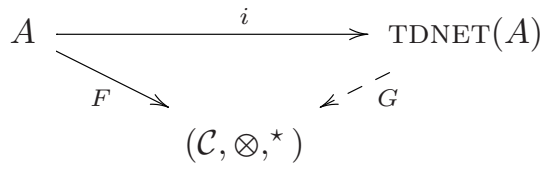

The proof of this theorem will be treated in Sections 6 through 9 .

\section{The virtual unit}

The natural way of modelling a proof net with a single port $A$ would be by a categorical map from the monoidal unit $I$ into $A$. Lacking units, tensor-dual categories generally cannot describe such proof nets, or nets that have subnets with a single port. However, adding a monoidal unit to a tensor-dual category would make it $\star$-autonomous. Thus, the challenge is to capture single-port proof nets in another way.

The approach proposed by Lamarche and Strassburger in (Lamarche and Straßburger, 2005), and deepened by Houston in (Houston, 2008), is to add a virtual unit, a construction that can be axiomatised to act like a unit, but is not itself an object in the tensor-dual category. The virtual unit $\mathbb{I}$ for a TD category $\mathcal{C}$ will be an object in the category $\mathrm{SET}^{\mathcal{C}}$ of set-valued functors from $\mathcal{C}$. The objects of this category may be seen as generalised $\mathcal{C}$-objects via the Yoneda Lemma, by which $\mathcal{C}$ embeds into $\mathrm{SET}^{\mathcal{C}}$ by the contravariant Yoneda functor $h^{-}$, defined on objects and morphisms as follows.

$$
h^{A}=\operatorname{hom}(A,-): \mathcal{C} \rightarrow \operatorname{SET} \quad h^{f: A \rightarrow B}=(-\circ f): \operatorname{hom}(B,-) \Rightarrow \operatorname{hom}(A,-)
$$

A proof net corresponding to a map $A \rightarrow B$ in $\mathcal{C}$ is represented in SET $^{\mathcal{C}}$ by a natural transformation $h^{B} \Rightarrow h^{A}$; similarly, II will be axiomatised such that a proof net for a sequent $\vdash B$, with a single port, is represented by a natural transformation $h^{B} \Rightarrow \mathbb{I}$. Via Yoneda, any such natural transformation is uniquely determined by an element $x$ of $\mathbb{I}(B)$. Although traditionally such transformations are denoted simply as $x$, here instead the notation $\mathbb{I}-(x)$ will be used, to emphasise their use as a syntactic construct in proof nets and sequent calculus presentations. The functor $\mathbb{I}$ may thus be seen as a unary hom-functor, taking an object $B$ to the set of proof nets for $\vdash B$.

In the functor category $\mathrm{SET}^{\mathcal{C}}$, the tensor of $\mathcal{C}$ may be analysed in terms of composition 


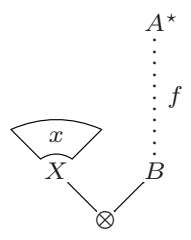

$x \otimes f$

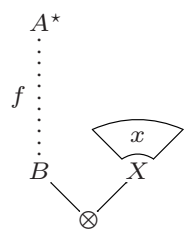

$f \otimes x$

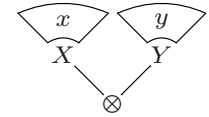

$x \otimes y$

Fig. 5. Virtual tensor constructions

with the internal hom-functor $H^{-}$, as given by

$$
H^{B}=\left(B \otimes-{ }^{\star}\right)^{\star} \stackrel{\partial}{=}\left(B^{\star} 8-\right),
$$

in the following way:

$$
h^{A \otimes B}=\operatorname{hom}(A \otimes B,-) \stackrel{\Phi}{\cong} \operatorname{hom}\left(A, B^{\star} 8-\right)=h^{A} \circ H^{B} .
$$

Remark. The above is a special case of the Day convolution tensor (Day, 1970). The general construction gives a tensor product in the functor category $\mathrm{SET}^{\mathcal{C}}$, by the following coend formula. For two functors $F, G: \mathcal{C} \rightarrow \mathrm{SET}$,

$$
F \star G=\int^{C, D \in \mathcal{C}} F C \times G D \times \operatorname{hom}(C \otimes D,-) .
$$

For $h^{A}$ and $h^{B}$ this gives:

$$
h^{A} \star h^{B}=\int^{C, D \in \mathcal{C}} \operatorname{hom}(C, A) \times \operatorname{hom}(D, B) \times \operatorname{hom}(C \otimes D,-) .
$$

This is isomorphic to $\operatorname{hom}(A \otimes B,-)$ by the co-Yoneda lemma, which gives:

$$
\int^{C} \operatorname{hom}(C,-) \times F C \cong F
$$

In order to make $\mathbb{I}$ a left unit to the tensor in $\mathrm{SET}^{\mathcal{C}}$, the following natural isomorphism $\lambda$ is needed:

$$
\lambda: \mathbb{I} \circ H^{-} \cong h^{-} \quad \lambda_{A}: \mathbb{I}\left(A^{\star} 8-\right) \cong \operatorname{hom}(A,-) .
$$

This is exactly what one would expect: in a free setting, $\mathbb{I}\left(A^{\star} \ngtr B\right)$ and $\operatorname{hom}(A, B)$ should both be (isomorphic to) the set of proof nets for the sequent $\vdash A^{\star}, B$. Naturality of $\lambda$ is illustrated in the following diagram.

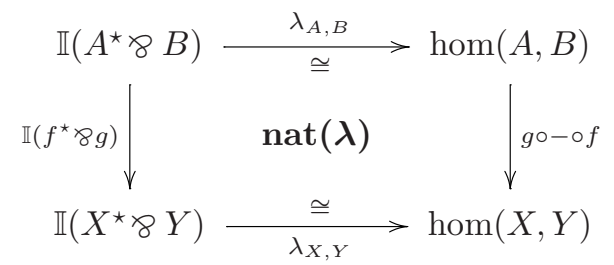

To represent proof nets with one-port subnets, it must be possible to combine proof nets for $\vdash X$ and $A \vdash B$ with a tensor, into a net for $A \vdash X \otimes B$. For this a virtual tensor 
operation is needed, taking $x \in \mathbb{I}(X)$ and $f: A \rightarrow B$ to

$$
x \otimes f: A \rightarrow X \otimes B .
$$

It can be defined via the Day tensor in $\mathrm{SET}^{\mathcal{C}}$, whose action on morphisms is horizontal composition of natural transformations. For $\mathbb{I}-(x): h^{X} \Rightarrow \mathbb{I}$ and $H^{f}: H^{B} \Rightarrow H^{A}$ there is the diagram below, with its composite natural transformation given below right.

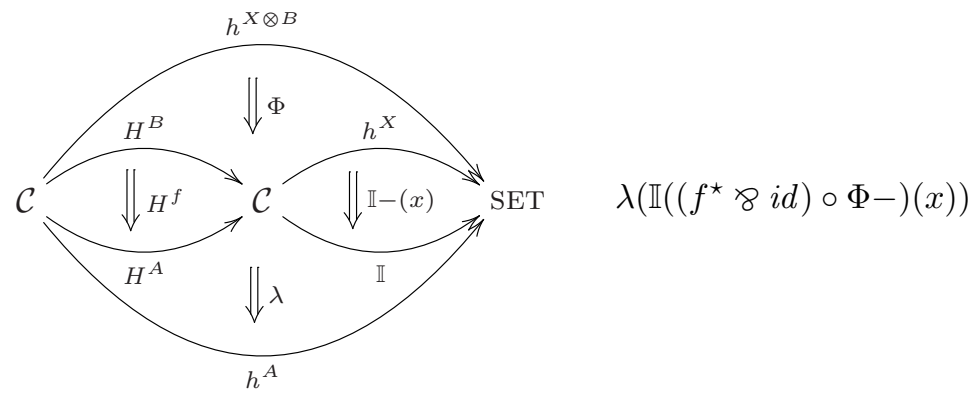

By Yoneda, this natural transformation gives a map $A \rightarrow X \otimes B$ when appied to $i d_{X \otimes B}$. The virtual tensor is then defined as follows (using naturality of $\lambda$ to increase readability).

$$
\begin{aligned}
& x \otimes f \triangleq \lambda\left(\mathbb{I}\left(\left(f^{\star} \ngtr i d\right) \circ \Phi(i d)\right)(x)\right) \\
& =\lambda\left(\mathbb{I}\left(\left(f^{\star} \varnothing i d\right) \circ \eta\right)(x)\right) \\
& =\lambda(\mathbb{I}(\eta)(x)) \circ f
\end{aligned}
$$

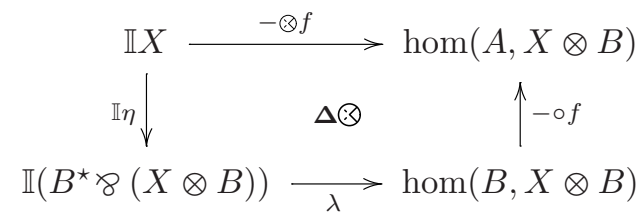

Two further virtual tensor operations can be derived from this: the first is a virtual tensor whose one-port argument is on the right, rather than on the left, obtained via symmetry, and the second combines two one-port arguments, $x \in \mathbb{I}(X)$ and $y \in \mathbb{I}(Y)$, and is illustrated by the diagram below.

$$
\begin{array}{lll}
f \otimes x \triangleq \sigma \circ(x \otimes f) & \mathbb{I} X \times \mathbb{I} Y \stackrel{(-\otimes i d) \times \mathbb{I} Y}{\longrightarrow} \operatorname{hom}(Y, X \otimes Y) \times \mathbb{I} Y \\
x \otimes y \triangleq \mathbb{I}\left(x \otimes i d_{Y}\right)(y) & -\otimes-\mid \begin{array}{l}
\Delta \otimes \\
\Delta
\end{array} & \mathbb{I}(X \otimes Y Y) \underset{\text { apply }}{\leftarrow} \operatorname{hom}(\mathbb{I} Y, \mathbb{I}(X \otimes Y)) \times \mathbb{I} Y
\end{array}
$$

How the three virtual tensors are used to interpret proof nets is illustrated in Figure 5. The present section is concluded by noting a central property of the virtual tensor.

Lemma 4.1. The diagram @e below commutes.

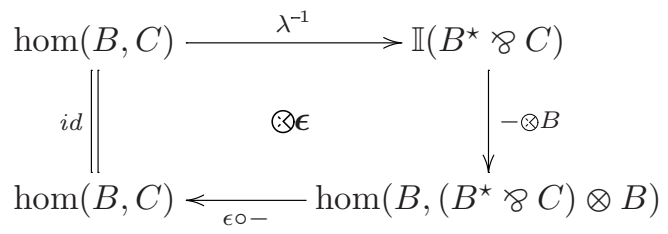

Proof. By the diagram below. The unmarked regions commute by invertibility and 

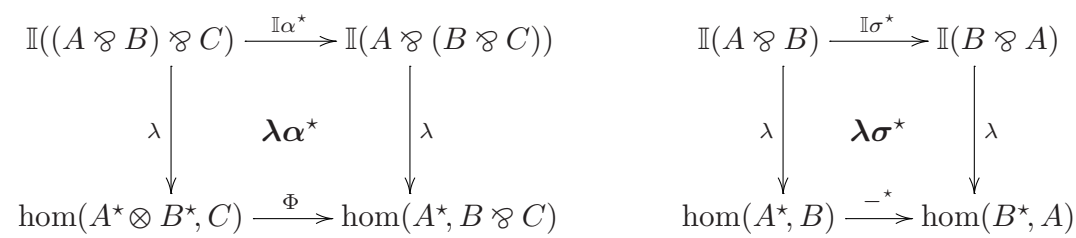

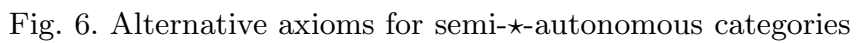

naturality of $\lambda$, and $\eta \epsilon$-cancellation.

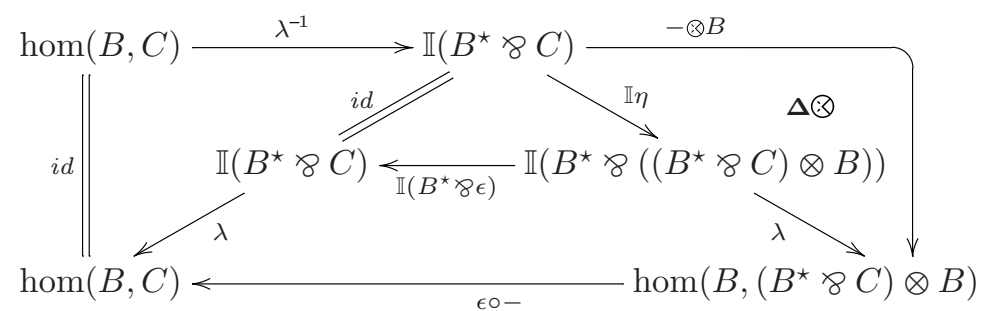

\section{Semi- $\star$-autonomous categories}

To establish the correspondence with proof nets, tensor-dual categories with a virtual unit need to satisfy the additional coherence axiom $\otimes \boldsymbol{\alpha}$, shown below. Corresponding diagrams in Houston's thesis (Houston, 2008) are (8.3.1) (a simplified diagram appears just below it) and $(\lambda \alpha \chi)$ (on page 187).

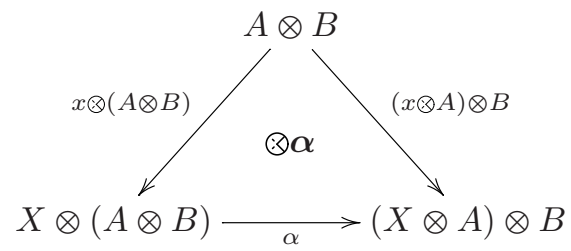

Definition 5.1 ((Lamarche and Straßburger, 2005, Definition 2.1.2), (Houston, 2008, Definition 8.8)). A semi- $\star$-autonomous (SSA) category $\left(\mathcal{C}, \otimes,{ }^{\star}, \mathbb{I}, \lambda\right)$ is a TD category $\left(\mathcal{C}, \otimes,{ }^{\star}\right)$ with a virtual unit functor $\mathbb{I}: \mathcal{C} \rightarrow$ SET and a natural isomorphism $\lambda_{A, B}: \mathbb{I}\left(A^{\star} \gamma B\right) \cong \operatorname{hom}_{\mathcal{C}}(A, B)$ such that the diagram $\otimes \boldsymbol{\alpha}$ commutes.

In Definition 2.1.2 of Lamarche and Strassburger (Lamarche and Straßburger, 2005), an additional axiom - equation (2) in that paper - is asked for. The corresponding diagram is $\circledast \sigma$ in Figure 8. Proposition 5.6 will show that it follows from the other axioms.

An alternative formulation of semi- -autonomous categories replaces the diagrams $\mathbf{\Phi} \boldsymbol{\alpha}$, $\boldsymbol{\Phi} \boldsymbol{\sigma}$, and $\otimes \boldsymbol{\alpha}$ with the diagrams $\boldsymbol{\lambda} \boldsymbol{\alpha}^{\star}$ and $\boldsymbol{\lambda} \boldsymbol{\sigma}^{\star}$ in Figure 6. Equivalence of both definitions is shown by Proposition 5.3 and Proposition 5.4 below. The two diagrams $\lambda \boldsymbol{\alpha}^{\star}$ and $\boldsymbol{\lambda} \boldsymbol{\sigma}^{\star}$ allow to omit the isomorphisms $\Phi$ and $\partial$ (respectively) from the data, instead defining them via $\mathbb{I} \alpha^{\star}$ and $\mathbb{I} \sigma^{\star}$. Doing so gives the following simplified definition of semi$\star$-autonomous categories: 
Proposition 5.2 ((Houston, 2008, Proposition 8.9)). An SSA-category $\mathcal{C}$ is characterised by an associative, symmetric tensor $\otimes$, an involutive duality ${ }^{\star}$, and a virtual unit functor $\mathbb{I}: \mathcal{C} \rightarrow$ SET with a natural isomorphism $\lambda_{A, B}: \mathbb{I}\left(A^{\star} \ngtr B\right) \cong \operatorname{hom}(A, B)$.

In the following, note that in larger diagrams an alternative, more concise notation is used.

$$
\begin{aligned}
& \bar{A}:=A^{\star} \quad A B \quad:=A \otimes B \\
& A, B:=A \ngtr B \quad A \triangleright B \quad:=\operatorname{hom}(A, B)
\end{aligned}
$$

Proposition 5.3 ((Houston, 2008, Lemmata 8.5-8.7)). In the definition of semi$\star$-autonomous categories, the axiom pair $\mathbf{\Phi} \boldsymbol{\alpha}$ and $\otimes \boldsymbol{\alpha}$ is interchangeable with the axiom $\lambda \boldsymbol{\alpha}^{\star}$, independently of the axiom $\boldsymbol{\Phi} \boldsymbol{\sigma}$.

While the Lemmata 8.5-8.7 In Houston's thesis (Houston, 2008) prove a moral equivalent of the above proposition, the corresponding axioms- $(\alpha \phi),(\lambda \alpha \chi)$, and $(\lambda \chi \psi)$-and the technical exposition are sufficiently different that proving the proposition here is worth wile.

Proof. Firstly, given $\boldsymbol{\lambda} \boldsymbol{\alpha}^{\star}$, the diagram $\boldsymbol{\Phi} \boldsymbol{\alpha}$ follows directly by applying $\lambda$ to each corner of the associativity pentagon for $\alpha^{\star}$. (The other direction is not immediate since the diagram just described uses multiple instances of $\left.\boldsymbol{\lambda} \boldsymbol{\alpha}^{\star}\right)$. The diagram below proves (3) from $\boldsymbol{\lambda} \boldsymbol{\alpha}^{\star}$ and $\boldsymbol{\Phi} \boldsymbol{\alpha}$, the latter of which is the circumference of the diagram.

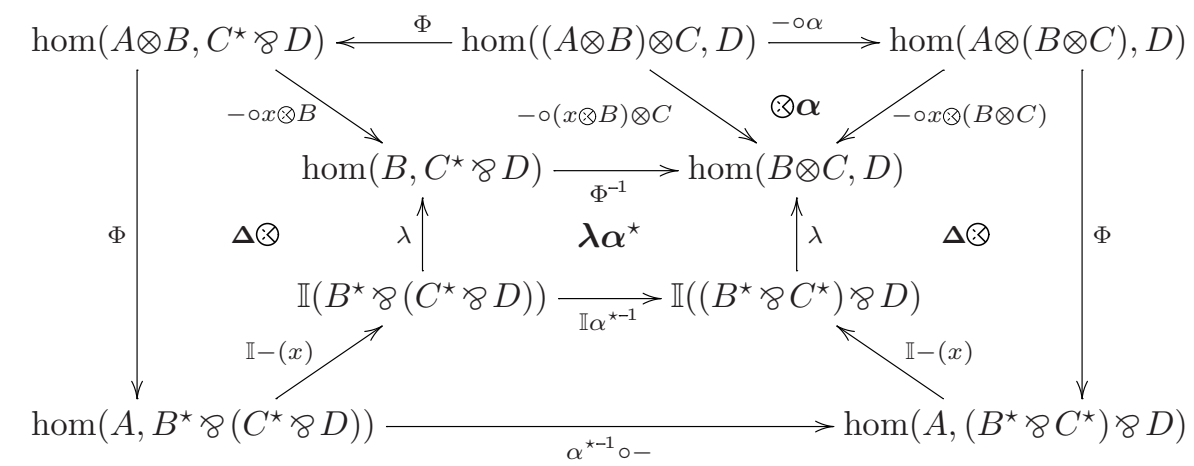

This proves one direction. The other direction, that $\boldsymbol{\lambda} \boldsymbol{\alpha}^{\star}$ follows from $\otimes \boldsymbol{\alpha}$ and $\mathbf{\Phi} \boldsymbol{\alpha}$, is proven by the first diagram in Figure 7 . The outside of the diagram commutes by functoriality of the tensor, and the unlabelled interior regions commute either trivially, by naturality, or by $\eta-\epsilon$ cancellation. The region marked (1) commutes by tracing $\mathbf{\Phi} \boldsymbol{\alpha}$, in the second and third diagram in Figure 7.

Proposition 5.4. In the definition of semi- $\star$-autonomous categories, the axiom $\boldsymbol{\Phi} \boldsymbol{\sigma}$ is interchangeable with the axiom $\lambda \boldsymbol{\sigma}^{\star}$.

Proof. In the diagram below, the outside is the diagram $\boldsymbol{\Phi} \boldsymbol{\sigma}$, while the far left region is $\lambda \boldsymbol{\sigma}^{\star}$. The central region is the hexagon for $\sigma^{\star}$ and $\alpha^{\star}$, and the two remaining unlabelled 

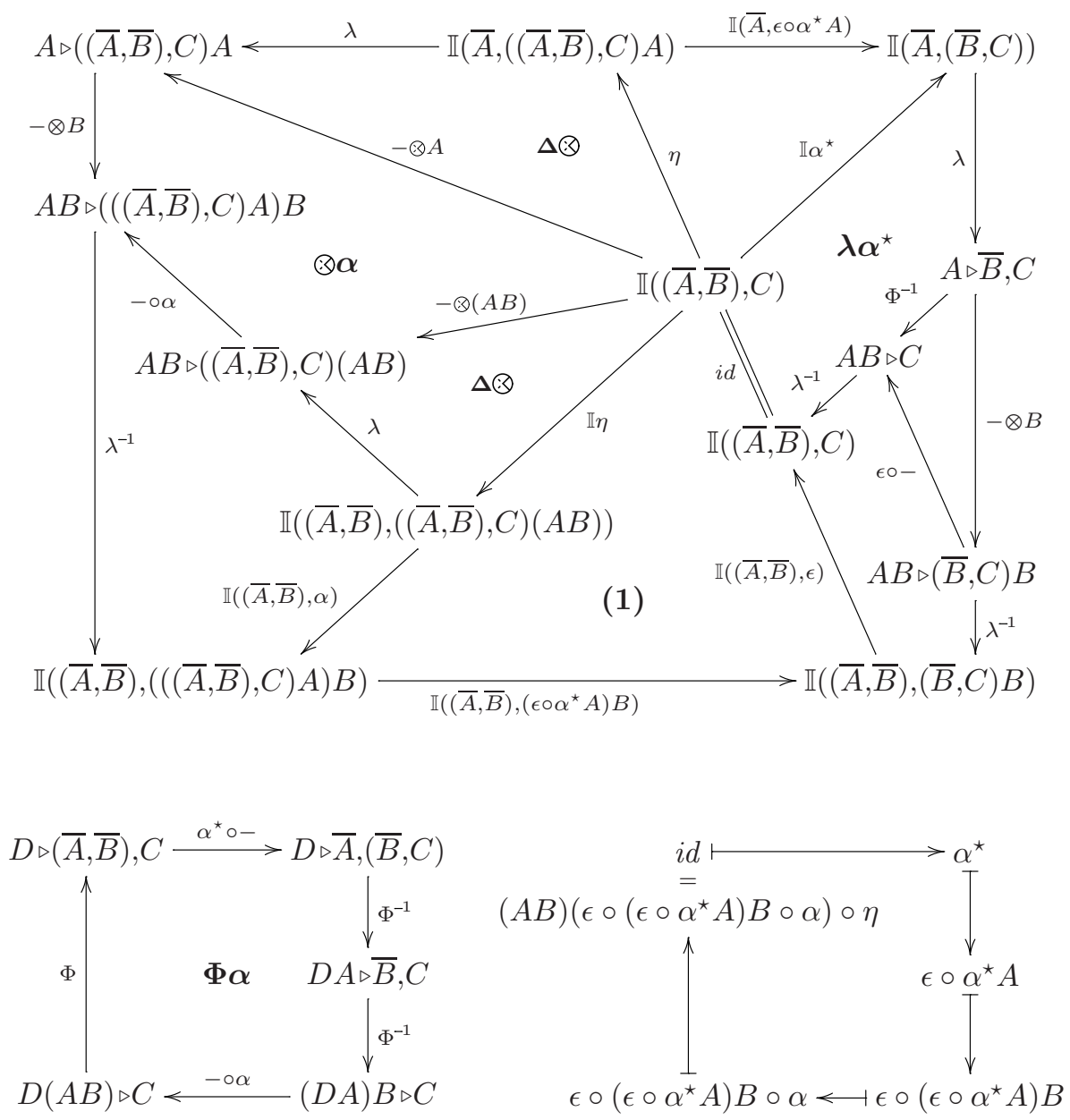

Fig. 7. Diagrams for Proposition 5.3

regions commute by naturality. This shows that $\boldsymbol{\Phi} \boldsymbol{\sigma}$ follows from $\lambda \boldsymbol{\sigma}^{\star}$.

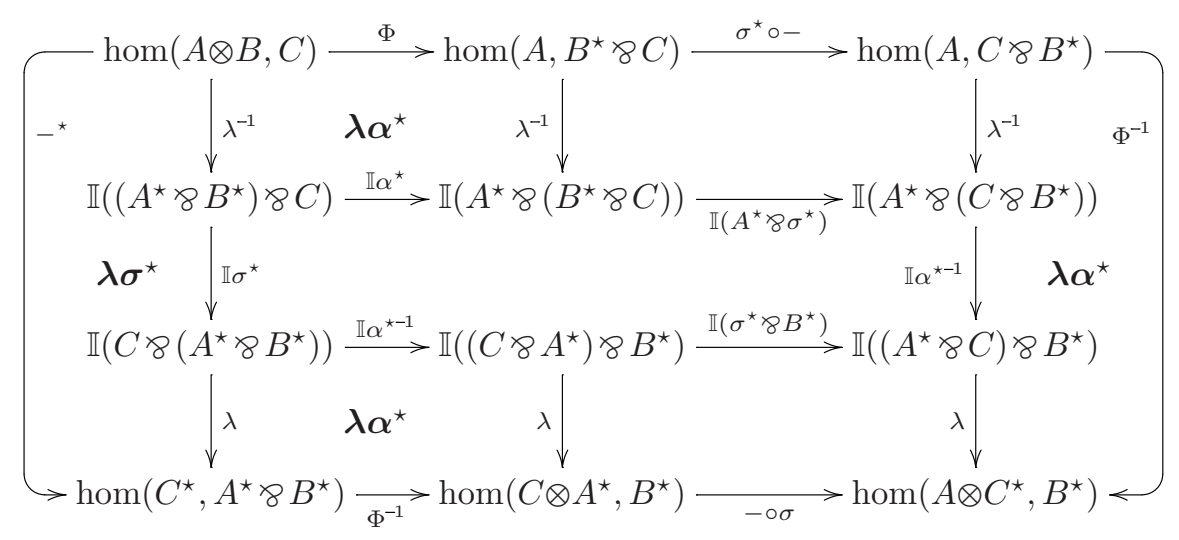



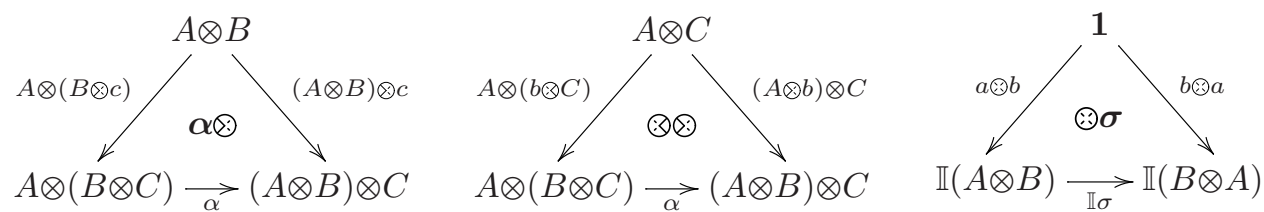

Fig. 8. Coherence diagrams for the virtual tensors

In the other direction, first let $f^{\perp}$ denote $\lambda\left(\mathbb{I} \sigma^{\star}\left(\lambda^{-1}(f)\right)\right)$; the diagram $\boldsymbol{\lambda} \boldsymbol{\sigma}^{\star}$ then expresses the equation $f^{\perp}=f^{\star}$. Since all arrows are invertible in the above diagram, it shows that $f^{\perp}=f^{\star}$ follows from $\boldsymbol{\Phi} \boldsymbol{\sigma}$ in the restricted case where $f: A \otimes B \rightarrow C$; in particular, $\epsilon^{\perp}=\epsilon^{\star}$. Secondly, the following diagram shows that $(g \circ f)^{\perp}=f^{\star} \circ g^{\perp}$; all three regions commute by naturality.

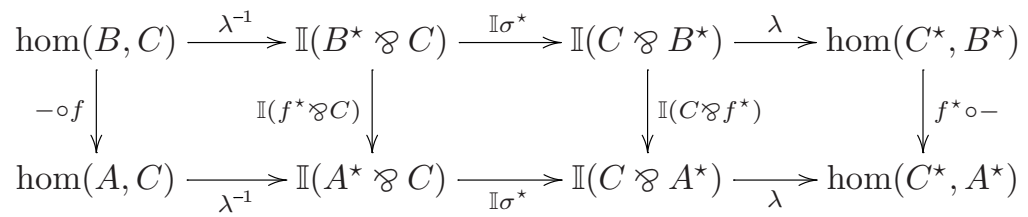

Using the equation $\otimes \epsilon$ in Lemma $4.1, f=\epsilon \circ\left(\lambda^{-1}(f) \otimes i d\right)$, there is the following series of equations, proving the statemement.

$$
\begin{aligned}
f^{\perp} & =\left(\epsilon \circ\left(\lambda^{-1}(f) \otimes i d\right)\right)^{\perp} \\
& =\left(\lambda^{-1}(f) \otimes i d\right)^{\star} \circ \epsilon^{\perp} \\
& =\left(\lambda^{-1}(f) \otimes i d\right)^{\star} \circ \epsilon^{\star} \\
& =\left(\epsilon \circ\left(\lambda^{-1}(f) \otimes i d\right)\right)^{\star}=f^{\star}
\end{aligned}
$$

\section{The virtual tensor}

It will be shown that the diagrams in Figure 8 commute (note that 1 denotes the terminal object in SET). That these appear structurally similar to the familiar diagrams for the monoidal unit and its left and right unit isomorphisms, is no coincidence, as will be explained next.

One difficulty with semi- $\star$-autonomous categories is that on first sight there appears to be no virtual equivalent to an object such as $I \otimes I$. However, the promonoidal perspective shows that there is one: it is given by the following coend formula.

$$
\int^{A, B} \mathbb{I} A \times \mathbb{I} B \times \operatorname{hom}(A \otimes B,-)
$$

This is a set-valued functor, taking an object $C$ to an equivalence class of triples

$$
(a \in \mathbb{I} A, b \in \mathbb{I} B, f: A \otimes B \rightarrow C \text { ) }
$$

— for details see Kelly's book (Kelly, 1982). In the present setting, such triples are in 

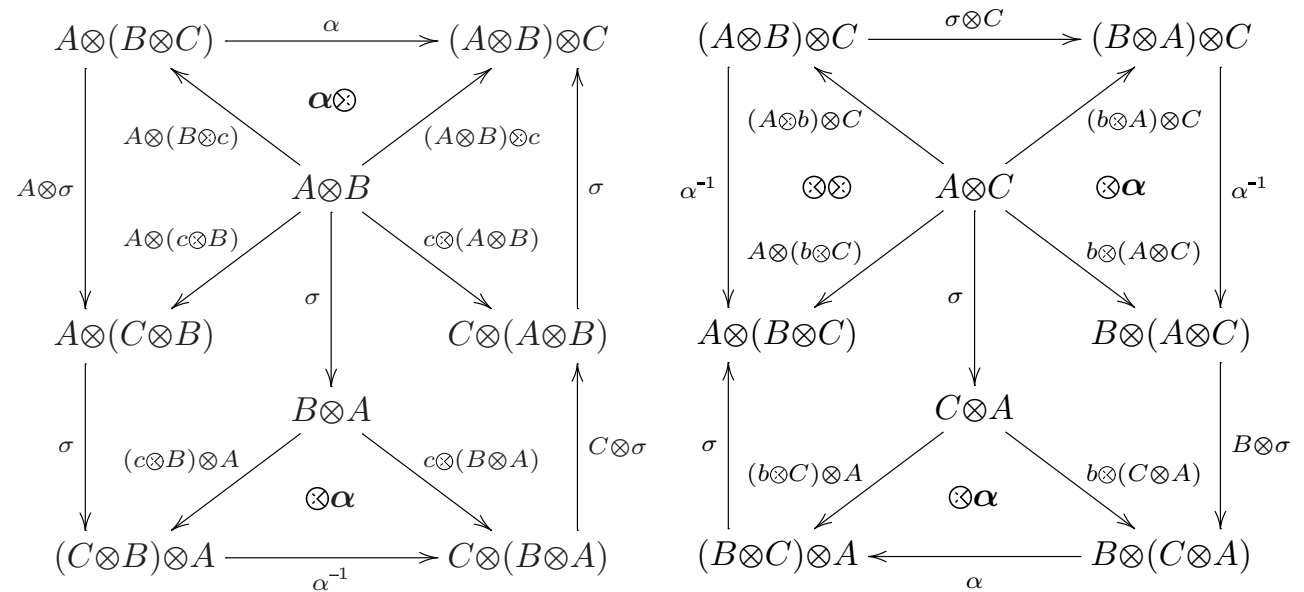

Fig. 9. Diagrams proving that $\boldsymbol{\alpha} \circledast$ and $\otimes \circledast$ commute

one-to-one correspondence with morphisms

$$
\mathbb{I} f(a \otimes b),
$$

where the practical consequence of the equivalence relation induced by the coend formula is that $a$ and $b$ (and $A$ and $B$ ) should be considered arbitrary.

All this is to say that for reasoning within semi- $\star$-autonomous categories, it is not $\lambda: \mathbb{I}\left(A^{\star} \ngtr B\right) \rightarrow \operatorname{hom}(A, B)$ that behaves most like a monoidal left unit isomorphism, but instead it is the virtual tensor,

$$
a \otimes B: B \rightarrow A \otimes B
$$

for arbitrary $a$ and $A$. Crucially, the virtual tensor is invertible, by taking the special case $a=\lambda^{-1}\left(i d_{B}\right)$ and composing with $\epsilon$ :

$$
\epsilon \circ\left(\lambda^{-1}\left(i d_{B}\right) \otimes B\right)=i d_{B} .
$$

The equation is a special case of the diagram @e of Lemma 4.1, which shows the more general case for $f: B \rightarrow C$ rather than $i d_{B}$. With this in mind, the diagrams in Figure 8 can be shown to commute by interpreting the standard coherence proofs for the monoidal unit, where the right-virtual tensor, $\ominus$, corresponds to the right unit isomorphism $\rho$.

Proposition 5.5. The diagrams $\boldsymbol{\alpha} \ominus$ and $\bigotimes \ominus$ in Figure 8 commute.

Proof. By the two diagrams in Figure 9. For both diagrams the outside commutes by $\alpha-\sigma$ coherence, while unlabelled internal regions commute by naturality or by the definition of $\otimes$.

The third diagram, $\odot \boldsymbol{\sigma}$, corresponds to the equation $\lambda_{I}=\rho_{I}$ in monoidal categories, 
illustrated below left.
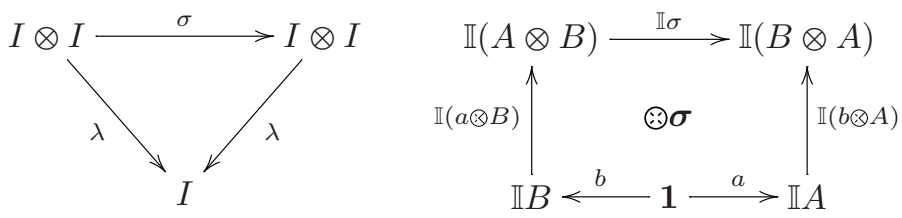

Shown above right, first the unit $I$ corresponds to an equivalence class over all virtual morphisms $a \in \mathbb{I} A$, for arbitrary $a$ and $A$. To close the diagram with a left-virtual tensor and a right-virtual tensor, in one case $a$ is chosen to represent this equivalence class, but in the other, $b$.

With this interpretation, the standard proof of $\lambda_{I}=\rho_{I}$ can be adapted to show $\circledast \sigma$. A useful ingredient here is a virtual equivalent to the morphism $\rho_{I} \circ \lambda_{I}^{-1}: I \rightarrow I$. For arbitrary $c \in \mathbb{I} C$, let the element " $c$ " $\in \mathbb{I} C$ be defined as "c" $=\mathbb{I}(\epsilon \circ \sigma)\left(c \otimes \lambda^{-1}\left(i d_{C}\right)\right)$, as in the following diagram.

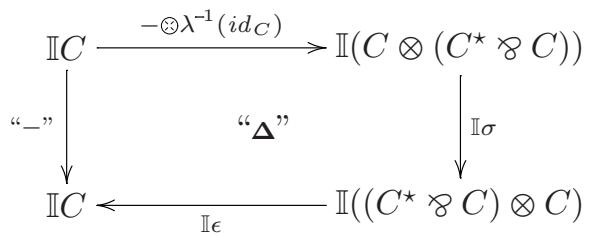

While the equation $\mathbb{I} \epsilon\left(\lambda^{-1}\left(i d_{C}\right) \otimes c\right)=c$ is immediate from $\otimes \epsilon$, to show " $c$ " $=c$ requires the symmetry on the virtual tensor. This will now be proved.

Proposition 5.6 (Due to Houston, in private communication). The diagram $\circledast \sigma$ in Figure 8 commutes.

Proof. The first diagram in Figure 10 shows the equation

$$
\mathbb{I} \sigma(c \otimes a)=a \otimes " c " .
$$

Its regions commute by naturality, $\eta \epsilon$-cancellation, and by the definition of the virtual tensor. In the second diagram, the two squares labelled (1) commute by the equation above, while the unlabelled regions commute by naturality of $\otimes$ and $\otimes$. The diagram gives the equation

$$
c \otimes(b \otimes a)=c \otimes \mathbb{I} \sigma(a \otimes b) .
$$

Then by choosing $c=\lambda^{-1}\left(i d_{B \otimes A}\right)$ and applying $\mathbb{I} \epsilon$, the statement follows by Lemma 4.1 (the diagram $\otimes \epsilon)$ :

$$
\begin{aligned}
(b \otimes a) & =\mathbb{I} \epsilon\left(\lambda^{-1}\left(i d_{B \otimes A}\right) \otimes(b \otimes a)\right) \\
& =\mathbb{I} \epsilon\left(\lambda^{-1}\left(i d_{B \otimes A}\right) \otimes \mathbb{I} \sigma(a \circledast b)\right)=\mathbb{I} \sigma(a \circledast b) .
\end{aligned}
$$

\section{The main theorem}

In Section 3 it was discussed how TD categories represent proof nets with at least two ports. The virtual unit allows SSA categories to capture also the proof nets with a single 

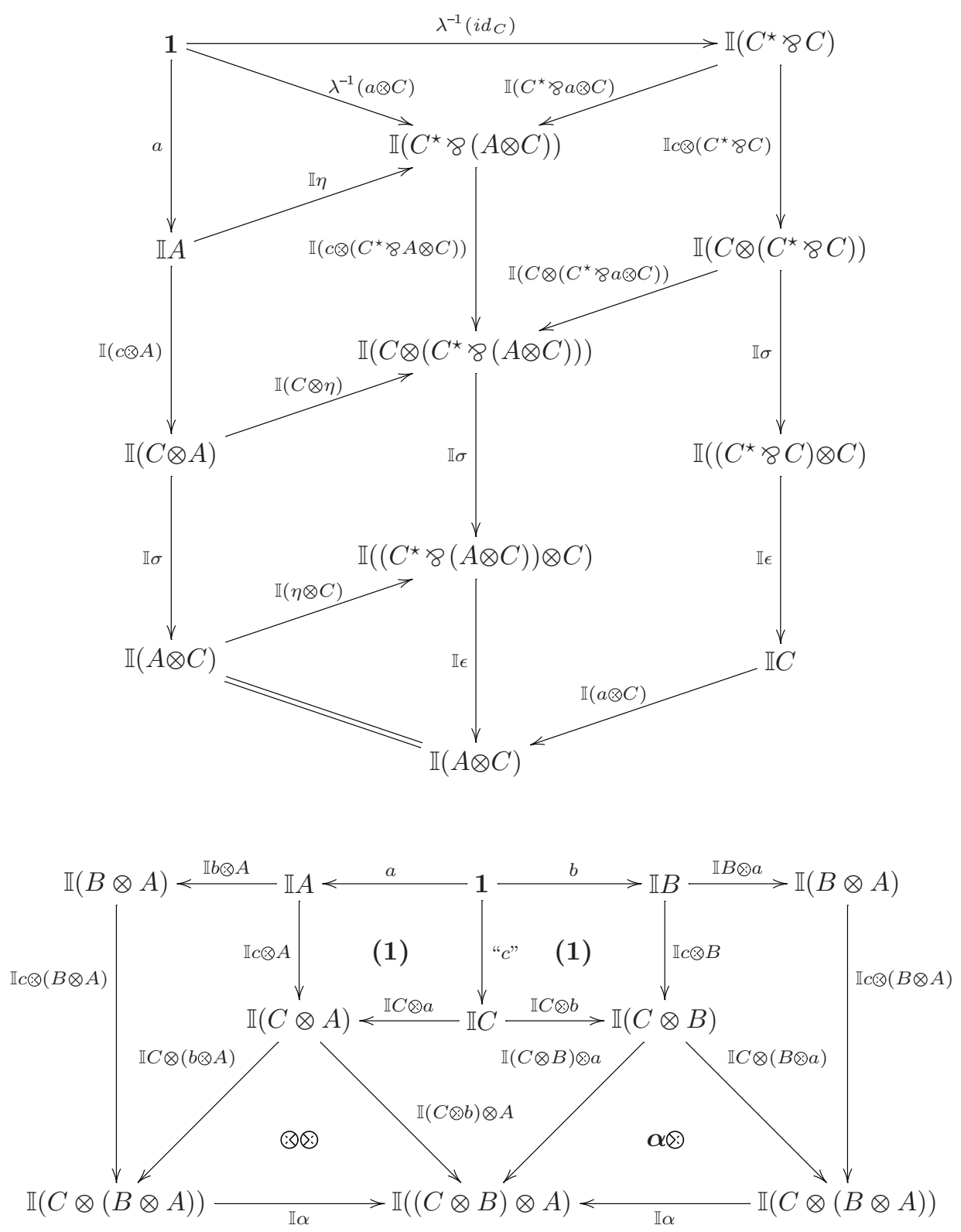

Fig. 10. Diagrams for Proposition 5.6 
port $A$, as the elements of $\mathbb{I}(A)$. On formulae of the form $A^{\star} \ngtr B$, the action of $\lambda$ is to remove the 8 -vertex at the root of a proof net for $\vdash A^{\star} \ngtr B$, as illustrated below; the inverse $\lambda^{-1}$ replaces it, similar to an application of a $\diamond \mathbf{R}$-inference to $\vdash A^{\star}, B$.

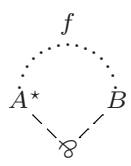

Then in the following let the category $\operatorname{NET}(\mathcal{A})$ be the category of proof nets $\mathcal{L}:\left[A_{V} \vdash B_{W}\right]$ and $\mathcal{L}:\left[\vdash A_{V}\right]$ over the atoms $\mathcal{A}$ (viewed as a discrete category).

Proposition 5.7. The category $\operatorname{NET}(\mathcal{A})$ is a semi- $\star$-autonomous category.

Proof. The illustrations in Figure 3 show how the category NET $(\mathcal{A})$ forms a TD-category. The isomorphism $\lambda$ is that taking a net $\mathcal{L}:\left[A^{\star} \ngtr B\right]$ to the net $\mathcal{L}:[A \vdash B]$, while $\mathbb{I}$ is the composition of a net $\mathcal{L}:\left[\vdash A_{V}\right]$ and one $\mathcal{K}:\left[A_{V} \vdash B_{W}\right]$ to form $\mathcal{L} ; \mathcal{K}:\left[\vdash B_{W}\right]$. The axiom $\odot \boldsymbol{\alpha}$ is then routinely verified.

It will be shown that $\operatorname{NET}(\mathcal{A})$ is, in fact, the free $\partial$-strict SSA category generated by $\mathcal{A}$. For making this precise, a notion of semi- $\star$-autonomous functor is needed. To preserve the semi- $\star$-autonomous structure, a functor $G$ between SSA categories $\mathcal{C}$ and $\mathcal{D}$ with virtual units $\mathbb{I}$ and $\mathbb{J}$ must not only take $\operatorname{hom}_{\mathcal{C}}(A, B)$ to $\operatorname{hom}_{\mathcal{D}}(G A, G B)$, but should in addition take $\mathbb{I}(A)$ to $\mathbb{J}(G A)$. It should thus come equipped with a natural transformation $\gamma: \mathbb{I} \Rightarrow \mathbb{J} G$. The combined ssa functor $(G, \gamma)$ must then preserve the virtual tensor construction, in the way illustrated below.

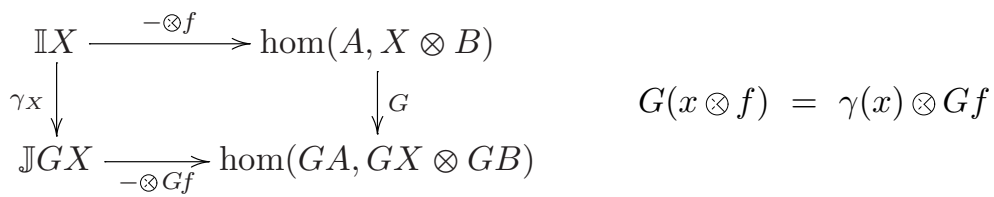

This is achieved by the following definition.

Definition 5.8. An SSA-functor is a pair

$$
(G, \gamma):\left(\mathcal{C}, \otimes_{\mathcal{C}},{ }^{\star}, \mathbb{I}, \lambda_{\mathcal{C}}\right) \rightarrow\left(\mathcal{D}, \otimes_{\mathcal{D}},{ }^{\star}, \mathbb{J}, \lambda_{\mathcal{D}}\right)
$$

such that $G: \mathcal{C} \rightarrow \mathcal{D}$ is a TD-functor and $\gamma: \mathbb{I} \rightarrow \mathbb{J} G$ is a natural transformation, satisfying the equation

$$
G(\lambda(x))=\lambda(\gamma(x)) .
$$

Proposition 5.9. The two conditions (2) and (3) are equivalent.

Proof. That (3) implies (2) follows simply by unfolding the definition of the virtual tensor; the other direction follows from the equation (هe) of Lemma 4.1, $f=\epsilon \circ\left(\lambda^{-1} f\right) \otimes i d$ :

$$
G(\lambda x)=G(\mathbb{I} \epsilon \circ(x \otimes i d))=\mathbb{J} G \epsilon \circ(\gamma(x) \otimes G i d)=\lambda(\gamma x) .
$$


The main theorem is then stated as follows, where $i: \mathcal{A} \rightarrow \operatorname{NET}(\mathcal{A})$ is the inclusion functor taking the atoms in $\mathcal{A}$ into $\operatorname{NET}(\mathcal{A})$.

Theorem 5.10. Let $F: \mathcal{A} \rightarrow \mathcal{C}$ be an arbitrary functor into a $\partial$-strict ssa category $\mathcal{C}$. Then there is a unique (up to natural isomorphism) ssa functor $(G, \gamma): \operatorname{NET}(\mathcal{A}) \rightarrow \mathcal{C}$ such that $F=G \circ i$.

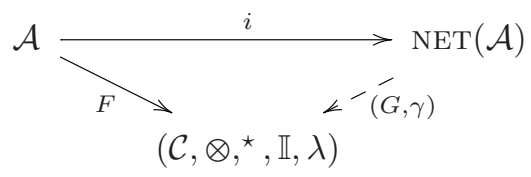

As before, this theorem may be seen as the coherence theorem for semi- $\star$-autonomous categories. The remainder of this paper will be devoted to proving it.

\section{A sequent calculus for categorical maps}

The one-sided sequents used for the sequent calculus constructing proof nets, in Figure 1, leave the structural isomorphisms $\alpha, \sigma, \Phi$ and $\lambda$ implicit. In general, a sequent calculus of this kind is underdetermined: it cannot distinguish, for instance, between the identity and the symmetry on $A \otimes A$. While the use of annotated formulae alleviates this problem, this section will present a sequent calculus that goes a step further, making all structural isomorphisms explicit. To this effect, first a notion of tree-sequent is introduced, an annotated sequent structured as a tree rather than a multiset, defined below left. To identify a subtree of such a tree, one-hole contexts are used, defined below right.

$$
t:=A_{V}|(t, t) \quad t\{-\}:=\{-\}|(t, t\{-\}) \mid(t\{-\}, t)
$$

A tree-context $t\{-\}$ is a tree-sequent containing a unique identifier $\{-\}$, the hole. Then $t\{s\}$ denotes the tree-sequent obtained by replacing the hole in $t\{-\}$ with a tree-sequent $s$. For readability, outer parentheses and vertex-annotation will be omitted where possible. The function $\lfloor-\rfloor$, defined below, retrieves the underlying annotated sequent of a treesequent $t$.

$$
\left\lfloor A_{V}\right\rfloor=\left\{A_{V}\right\} \quad\lfloor(s, t)\rfloor=\lfloor s\rfloor \uplus\lfloor t\rfloor
$$

Here, $\uplus$ denotes multiset union; we will require that $\lfloor t\rfloor$ is an annotated sequent, so that vertices in $t$ are unique. A tree-sequent $t$ also indicates an $\mathrm{MLL}^{-}$-formula, denoted as $\otimes t$, obtained by changing the formal tree $t$ into a tree of tensors, and forgetting the annotation of its leaves.

$$
\otimes\left(A_{V}\right)=A \quad \otimes(s, t)=(\otimes s) \otimes(\otimes t)
$$

The dual of a tree $t^{\star}$ is obtained by dualising its leaves, via $(s, t)^{\star}=\left(s^{\star}, t^{\star}\right)$. Then let $8 t$ denote $\left(\otimes t^{\star}\right)^{\star}$.

The purpose of tree-sequents is to facilitate the explicit treatment of the structural isomorphisms. The isomorphisms $\alpha$ and $\sigma$ can be applied 'deeply', i.e. within a given context, by inserting them into the hole of a tree-context $t\{-\}$, giving $t\{\alpha\}$ or $t\{\sigma\}$. The notation $\otimes t\{\alpha\}$ and $\otimes t\{\sigma\}$ then indicates the corresponding morphisms, as illustrated 
for $\otimes t\{\sigma\}$ below.

$$
\begin{aligned}
\otimes t\{\sigma\}: \otimes t\{(r, s)\} \rightarrow \otimes t\{(s, r)\} \quad & \otimes(s, t\{\sigma\})=\left(i d_{\otimes s}\right) \otimes(\otimes t\{\sigma\}) \\
\otimes(t\{\sigma\}, s) & =(\otimes t\{\sigma\}) \otimes\left(i d_{\otimes s}\right)
\end{aligned}
$$

To make $\Phi$ and $\lambda$ explicit a notion of two-sided tree-sequent is needed, written as $s \bullet t$, where $s$ and $t$ are tree-sequents. A two-sided sequent with empty antecedent is written $\bullet$. We write $s ? t$ to denote a tree-sequent with a possibly empty antecedent. A tree-sequent $s ? t$ has an underlying annotated sequent $\lfloor s ? \bullet t\rfloor$, and indicates a hom-set or virtual unit object $\operatorname{hom}(s ? t)$, as defined below.

$$
\begin{aligned}
\lfloor\bullet t\rfloor & =\lfloor t\rfloor & \operatorname{hom}(t) & =\mathbb{I}(\text { >t) } \\
\lfloor s \bullet t\rfloor & =\left\lfloor s^{\star}\right\rfloor \uplus\lfloor t\rfloor & \operatorname{hom}(s \bullet t) & =\operatorname{hom}(\otimes s, \text { ४t) }
\end{aligned}
$$

The structured sequent calculus LT, in Figure 11, employs two-sided tree-sequents to make the structural isomorphisms of semi- $\star$-autonomous categories explicit. This calculus serves as an intermediate between proof nets and categorical maps. On the one hand, each proof $\pi$ directly constructs a morphism in an arbitrary semi- $\star$-autonomous category $\mathcal{C}$ :

Definition 6.1. Given a functor $F: \mathcal{A} \rightarrow \mathcal{C}$, the denotation $(\pi)_{F}$ in $\mathcal{C}$ of an LT-proof $\pi$ is the $\mathcal{C}$-morphism it constructs. Two LT-proofs $\pi$ and $\rho$ are parallel if they have the same conclusion, and they are equivalent if they have the same denotation, i.e., $(\pi)_{F}=(\rho)_{F}$ for every $F$ and $\mathcal{C}$.

Here, the functor $F: \mathcal{A} \rightarrow \mathcal{C}$ is the interpretation of the atoms in $\mathcal{A}$ as objects in $\mathcal{C}$-note that in the calculus (in Figure 11) the identity axiom for an atomic $\mathrm{MLL}^{-}$formula $a$ constructs the identity map on $F a$.

On the other hand, by forgetting the tree-structure of its tree-sequents, every structured proof $\pi$ indicates a proof in the regular calculus LM, denoted $\lfloor\pi\rfloor$, which in turn constructs the proof net $\llbracket\lfloor\pi\rfloor \rrbracket$. The translation from LT-proofs to LM-proofs is a straightforward induction,

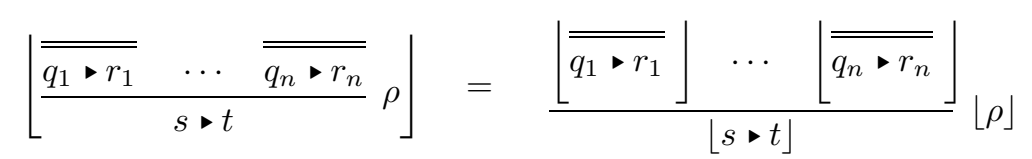

where the translation of an inference $\rho$ is given by the table below. Note that each LTinference maps onto one LM-inference with the exception of structural isomorphisms, which are implicit in LM. Note that as with LM-proofs, a double line will indicate a derivation consisting of zero, one, or multiple inferences, from multiple premisses. 
Logical rules

\begin{tabular}{|c|c|}
\hline${\overline{a_{v} \triangleright a_{w}}}^{i d_{F a}}$ & $\frac{\bullet A_{V} \quad t \bullet B_{W}}{t \bullet A_{V} \otimes_{u} B_{W}}$ \\
\hline & $s \bullet A_{V} \quad \neg B_{W}$ \\
\hline$s \bullet A_{V} \quad t \bullet B_{W}$ & $s \bullet A_{V} \otimes_{u} B_{W}$ \\
\hline$\overline{(s, t) \bullet A_{V} \otimes_{u} B_{W}}$ & - $A_{V} \quad \bullet B_{W}$ \\
\hline & - $A_{V} \otimes_{u} B_{W}$ \\
\hline$\frac{s \triangleright t\left\{A_{V}, B_{W}\right\}}{s \triangleright t\left\{A_{V} 8_{u} B_{W}\right\}}=$ & $\frac{\bullet A_{V}, B_{W}}{-A_{V} 8_{u} B_{W}}=$ \\
\hline
\end{tabular}

Structural isomorphisms

$$
\begin{aligned}
& \frac{(r, s) \bullet t}{r \bullet\left(s^{\star}, t\right)} \Phi \quad \frac{r \bullet(s, t)}{\left(r, s^{\star}\right) \bullet t} \Phi^{-1} \quad \frac{s \bullet t}{t^{\star} \bullet s^{\star}}-^{\star} \quad \mid \frac{\bullet(s, t)}{s^{\star} \bullet t} \lambda \quad \frac{s \bullet t}{\bullet\left(s^{\star}, t\right)} \lambda^{-1} \\
& \left.\frac{s\{((p, q), r)\} \bullet t}{s\{(p,(q, r))\} \bullet t}-\circ(\otimes s\{\alpha\}) \quad \frac{s \bullet t\{((p, q), r)\}}{s \bullet t\{(p,(q, r))\}}\left(8_{t}\left\{\alpha^{\star}\right\}\right) \circ-\quad \frac{\bullet t\{((p, q), r)\}}{\bullet t\{(p,(q, r))\}} \mathbb{I}\left(8_{t} t \alpha^{\star}\right\}\right) \\
& \left.\frac{s\{(p,(q, r))\} \bullet t}{s\{((p, q), r)\} \bullet t}-\circ\left(\otimes s\left\{\alpha^{-1}\right\}\right) \frac{s \bullet t\{(p,(q, r))\}}{s \bullet t\{((p, q), r)\}}\left(8_{t}\left\{\alpha^{\star-}\right\}\right) \circ-\frac{\bullet t\{(p,(q, r))\}}{\bullet t\{((p, q), r)\}} \mathbb{I}\left(8_{t} t \alpha^{\star-}\right\}\right)
\end{aligned}
$$

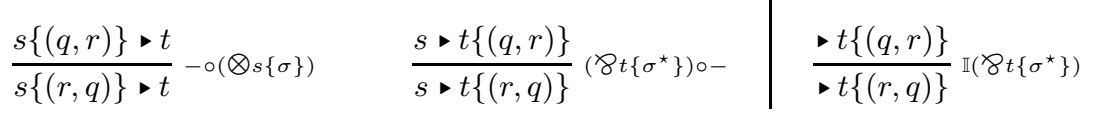

\section{Composition}

$$
\frac{s \bullet A_{V} A_{V} \bullet t}{s \bullet t} \circ \quad \mid \frac{\bullet A_{V} A_{V} \bullet t}{\bullet t} \mathbb{I}
$$

Fig. 11. The structured sequent calculus LT 


\begin{tabular}{cc}
$\rho$ & $\lfloor\rho\rfloor$ \\
\hline$i d_{F a}$ & $\mathbf{A X}$ \\
$\otimes, \otimes, \otimes, \otimes$ & $\otimes \mathbf{R}$ \\
$=$ & $\wp \mathbf{R}$ \\
$\circ, \mathbb{I}$ & $\mathbf{C u t}$ \\
structural isomorphism & $\mathrm{n} / \mathrm{a}$
\end{tabular}

The SSA-structure of the category of proof nets is nicely made explicit by using LTproofs to construct morphisms in $\operatorname{NET}(\mathcal{A})$ : for the inclusion functor $i: \mathcal{A} \rightarrow \operatorname{NET}(\mathcal{A})$, the denotation $(\pi)_{i}$ of a proof $\pi$ in $\operatorname{NET}(\mathcal{A})$ is the net constructed by it,

$$
(\pi)_{i}=\llbracket\lfloor\pi\rfloor \rrbracket .
$$

\section{Proving the main theorem}

Proving the main theorem requires to construct the SSA-functor $(G, \gamma)$ in the diagram repeated below.

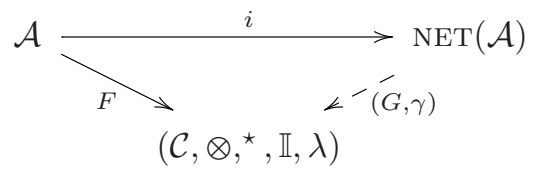

It will be constructed from three parts: a function $G_{0}$ on objects, a relation $G_{1}$ on morphisms, and a relation $\gamma$ on virtual unit morphisms. Define $G_{0}$ by

$$
\begin{aligned}
& G_{0} a=F a \quad G_{0}(A \otimes B)=G_{0} A \otimes G_{0} B \\
& G_{0} a^{\star}=(F a)^{\star} \quad G_{0}(A \ngtr B)=G_{0} A \ngtr G_{0} B \text {, }
\end{aligned}
$$

and let $G_{1}$ and $\gamma$ be given by the following composite arrow (in the category of sets and relations).

$$
\operatorname{NET}(A) \stackrel{\llbracket-\rrbracket^{-1}}{\longrightarrow} \mathrm{LM} \stackrel{L^{-\rfloor^{-1}}}{\longrightarrow} \mathrm{LT} \stackrel{\|^{-\nu_{F}}}{\longrightarrow} \mathcal{C}
$$

Thus, $G_{1}$ and $\gamma$ each take a net $N$ (with 2 ports respectively 1 port) to the set

$$
\left\{(0 \pi)_{F} \mid \llbracket\lfloor\pi\rfloor \rrbracket=N\right\} .
$$

In the sections to come it will be shown that $G_{1}$ and $\gamma$ are representable by functions (Proposition 9.2). Anticipating this fact, the proof of the main theorem is completed below.

Proof of Theorem 5.10 Firstly, by Proposition $9.2(G, \gamma)$ is a function on morphisms and virtual morphisms (that it is functional on objects is immediate). Secondly, given an LT-proof $\pi$, by definition $(G, \gamma)$ takes $\llbracket\lfloor\pi\rfloor \rrbracket$ to $(\pi)_{F}$. Since $\llbracket\lfloor\pi\rfloor \rrbracket=(\pi)_{i}$, this means that $(G, \gamma)$ takes $(\pi)_{i}$ to $(\pi)_{F}$. These two points suffice to make $(G, \gamma)$ an SSA-functor, since the calculus LT captures precisely the structure that must be preserved. Next, let $(H, \theta): \operatorname{NET}(\mathcal{A}) \rightarrow \mathcal{C}$ be an arbitrary sSA-functor such that $F=H \circ i$. For a given LTproof $\pi$, that $(H, \theta)$ preserves SSA-structure means it must take a proof net $(\pi)_{i}$ to a map 
canonically isomorphic to $(\pi)_{F}$. Since $(G, \gamma)$ takes $(\pi)_{i}$ to $(\pi)_{F}$, it follows that $(H, \theta)$ and $(G, \gamma)$ are canonically isomorphic.

A proof of Theorem 3.3, the corresponding theorem for tensor-dual categories, is contained within the proof of the main theorem, and essentially consists of a selection of the cases treated there. Those cases relevant to the tensor-dual case will be highlighted throughout the coming sections, and will be proven without making use of $\mathbb{I}$ and $\lambda$.

\section{Equivariance}

The first step in proving the main theorem will be a coherence result for the structural rules of the calculus LT. These rules are exactly what is made explicit in LT, compared to LM, and they allow to alternate between different tree-sequents with the same underlying annotated sequent. A sequence of structural rules will be called a structural derivation. The coherence result of this section will be that if two structural derivations have the same premiss and the same conclusion, they have the same categorical denotation.

Definition 7.1. Two tree-sequents $s ? \bullet t$ and $q$ ? $\bullet r$ are equivariant if they have the same underlying annotated sequent, $\lfloor s ? \bullet t\rfloor=\lfloor q$ ? $r\rfloor$. An equivariance isomorphism $\nu$ is a natural isomorphism in SET constructed by a structural derivation in LT,

$$
\frac{q ? \bullet r}{s ? \bullet t} \nu
$$

Extending the terminology for proofs, two structural derivations are parallel if they share the same premiss and conclusion, and equivalent if they construct the same equivariance isomorphism. The following is a generalisation of coherence for $\alpha$ and $\sigma$ (see (Mac Lane, 1963)), but weaker than full coherence for TD-categories or SSA-categories.

Proposition 7.2. Any two parallel structural derivations

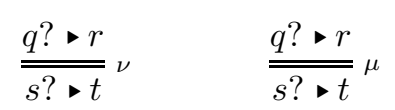

are equivalent, i.e. $\nu=\mu$.

The proof is standard, but included for completeness. In diagrams, coherence isomorphisms for $\alpha$ and $\sigma$ may be drawn as double lines, and left unlabelled.

Proof. Let us call structural inferences over $\alpha$ or $\sigma$ auxiliary, and those of the following kinds atomic:

$$
\frac{\left(r, A_{V}\right) \bullet t}{r \bullet\left(A_{V}^{\star}, t\right)} \Phi \quad \frac{r \bullet\left(A_{V}, t\right)}{\left(r, A_{V}^{\star}\right) \bullet t} \Phi^{-1} \quad \frac{A_{V} \bullet B_{W}}{B_{W}^{\star} \bullet A_{V}^{\star}}-^{\star} \quad \frac{\bullet\left(A_{V}, t\right)}{A_{V}^{\star} \bullet t} \lambda \quad \frac{A_{V} \bullet t}{\bullet\left(A_{V}^{\star}, t\right)} \lambda^{-1}
$$

i.e., in an atomic inference only single formulae are allowed to change sides. Let an auxiliary derivation be one over only auxiliary inferences, and an atomic derivation be one that may also include atomic inferences. Let the distance between two equivariant tree-sequents be the number of annotated formulae that are on opposite sides of the separator; e.g. $A_{V}$ counts for 1 but $B_{W}$ for 0 towards the distance between $\left.s \bullet t\left(A_{V}, B_{W}\right)\right\}$ 

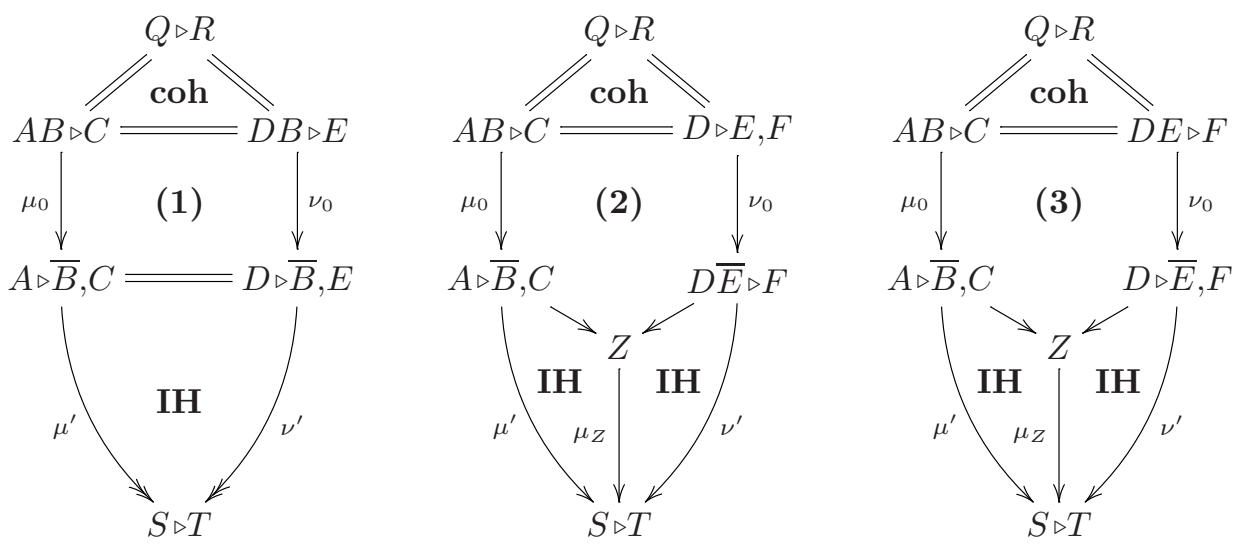

Fig. 12. Diagrams for equivariance I

and $q\left\{A_{V}^{\star}\right\} \bullet r\left\{B_{W}\right\}$. Let the distance of an equivariance derivation be that between its premiss and conclusion, and call a derivation decreasing if it has no subderivations of equal or greater distance. Note that between any two equivariant tree-sequents there is at least one atomic, decreasing derivation.

Narrowing down the possibilities, firstly, by the diagrams $\boldsymbol{\Phi} \boldsymbol{\alpha}, \boldsymbol{\Phi} \boldsymbol{\sigma}, \boldsymbol{\lambda} \boldsymbol{\alpha}^{\star}$, and $\boldsymbol{\lambda} \boldsymbol{\sigma}^{\star}$ each equivariance derivation is equivalent to an atomic one. Secondly, only tree-sequents with three or more annotated formulae need be considered: for sequents containing just two formulae the structural inferences that apply are $\lambda, \lambda^{-1},{ }^{\star}$ and $\mathbb{I} \sigma^{\star}$, whose only non-trivial interactions are described by the diagram $\lambda \boldsymbol{\sigma}^{\star}$. In particular, this means the inference rule - ${ }^{\star}$ may be ignored, since it is only atomic for tree-sequents $A_{V} \rightarrow B_{W}$, with single-formula antecedent and consequent. Thirdly, $\lambda$ - and $\lambda^{-1}$-inferences may be ignored: by $\boldsymbol{\lambda} \boldsymbol{\alpha}^{\star}$ consecutive $\lambda$ - and $\lambda^{-1}$-inferences may be replaced by $\Phi$ - and $\Phi^{-1}$-inferences, and two derivations starting (or ending) with a one-sided sequent $\bullet t$ have equivalent derivations starting (resp. ending) with the same $\lambda$-inference (resp. $\lambda^{-1}$-inference).

Next, it is shown that two atomic, decreasing derivations $\mu$ and $\nu$, not involving $-^{\star}$, $\lambda$, or $\lambda^{-1}$, are equivalent. The proof proceeds by induction on the distance of derivations. Let $\mu_{0}$ and $\nu_{0}$ be the first atomic inferences in $\mu$ and $\nu$, respectively. There are three cases.

- Both $\mu_{0}$ and $\nu_{0}$ move $B_{V}$ from left to right (or from right to left),

- $\mu_{0}$ moves $B_{V}$ from left to right, while $\nu_{0}$ moves $E_{W}$ from right to left,

- $\mu_{0}$ and $\nu_{0}$ respectively move $B_{V}$ and $E_{W}$ from left to right (or from right to left).

These cases correspond to the three diagrams shown in Figure 12. In the first case, the upper triangle commutes by coherence of symmetric monoidal categories; note that $A$ and $D$ are $\alpha \sigma$-isomorphic, as are $C$ and $E$. The rectangle (1) commutes by naturality of $\Phi$, and the lower triangle commutes by the induction hypothesis. In the second case, again the upper triangle commutes by coherence, while the lower two lozenges commute by the induction hypothesis. The central pentagon (2) commutes by the first diagram in Figure 13, modulo coherence in monoidal categories. In the third case, there are two 
cases for the central pentagon (3): when there are more than two objects on the left, and when there are exactly two objects on the left. The first is again covered by the first diagram in Figure 13, this time read from left to right instead of from top to bottom. The second case is covered by the second diagram, read from left to right (or from top right to bottom left, or from bottom right to top left).

Finally, it will be shown that, given the above, every derivation is equivalent to a decreasing one. The argument will assume a longest subderivation with a decreasing equivalent, and show by contradiction that it must encompass the entire derivation. Given a derivation with target $s ? t$, let $\mu$ be its longest subderivation, including the target $s ? \bullet t$, that is equivalent to a decreasing derivation - for simplicity, assume that $\mu$ itself is decreasing. If an atomic inference $\mu_{0}$ precedes $\mu$, as below left, then there is a decreasing derivation $\nu \circ \mu_{0}^{-1}$ as below right.

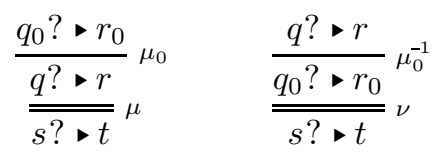

But if parallel decreasing derivations are equivalent, $\mu$ is equivalent to $\nu \circ \mu_{0}^{-1}$, and $\mu \circ \mu_{0}$ must be equivalent to $\nu$. As $\nu$ is decreasing, this contradicts the assumption that $\mu$ is the longest subderivation with a decreasing equivalent; hence, there can be no $\mu_{0}$, and $\mu$ must be the whole derivation.

Combining the above, for any two parallel derivations there are equivalent decreasing derivations, which must then be equivalent, proving the statement.

Proposition 7.3. Let $\pi$ and $\rho$ be two parallel LT-proofs. If $\lfloor\pi\rfloor=\lfloor\rho\rfloor$ then $\pi$ and $\rho$ are equivalent, i.e., $(\pi)_{F}=(\rho)_{F}$ for any $F$.

Proof. By induction on $\lfloor\pi\rfloor=\lfloor\rho\rfloor$. One case, illustrated below, is treated explicitly; the other cases are similar. Let $\lfloor\pi\rfloor=\lfloor\rho\rfloor, \pi$, and $\rho$ be respectively:

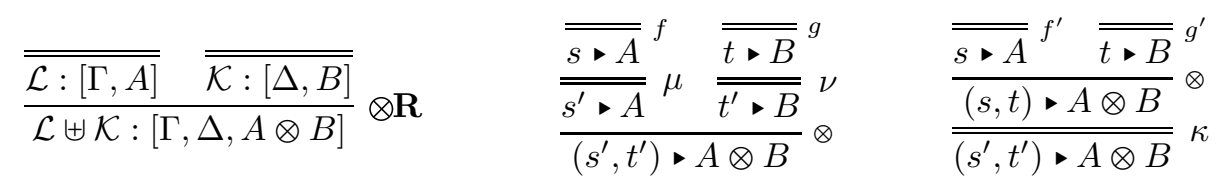

(Note that, without loss of generality, possible equivariance inferences at the end of $\pi$ and $\rho$ are ignored.) By the induction hypothesis $f=f^{\prime}$ and $g=g^{\prime}$. Since $\lfloor s\rfloor=\left\lfloor s^{\prime}\right\rfloor$ and $\lfloor t\rfloor=\left\lfloor t^{\prime}\right\rfloor$, by Proposition 7.2 the equivariance isomorphisms $\mu$ and $\nu$ are of the form $-\circ k$ and $-\circ k^{\prime}$ respectively, where $k$ and $k^{\prime}$ are composed over $\alpha, \sigma$ and inverses. Then by the same proposition $\kappa=-\circ k \otimes k^{\prime}$, and

$$
(f \circ k) \otimes\left(g \circ k^{\prime}\right)=(f \otimes g) \circ\left(k \otimes k^{\prime}\right)=\left(f^{\prime} \otimes g^{\prime}\right) \circ \kappa .
$$




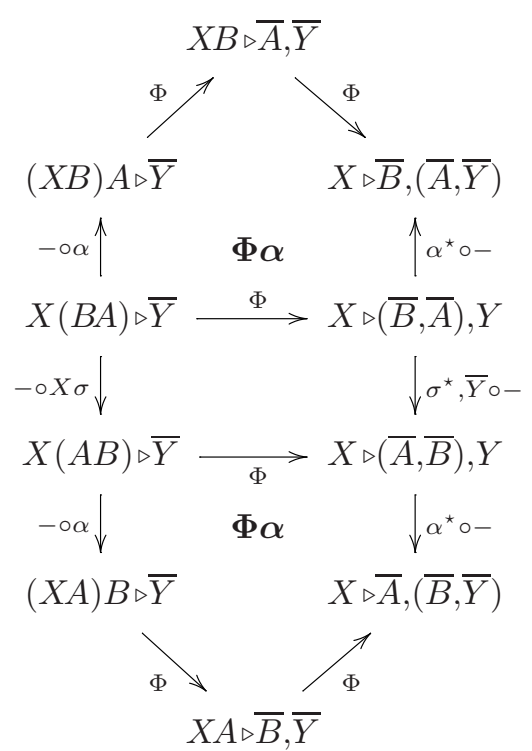

$\Phi \alpha 2$

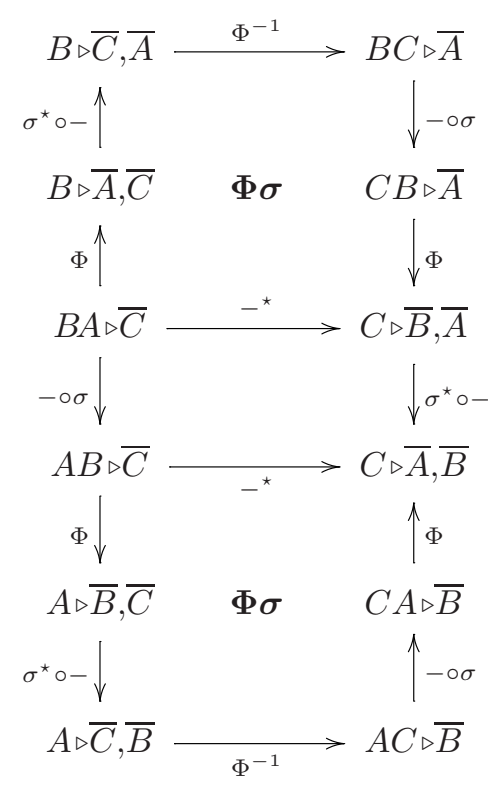

$\Phi \sigma 2$

Fig. 13. Diagrams for equivariance II

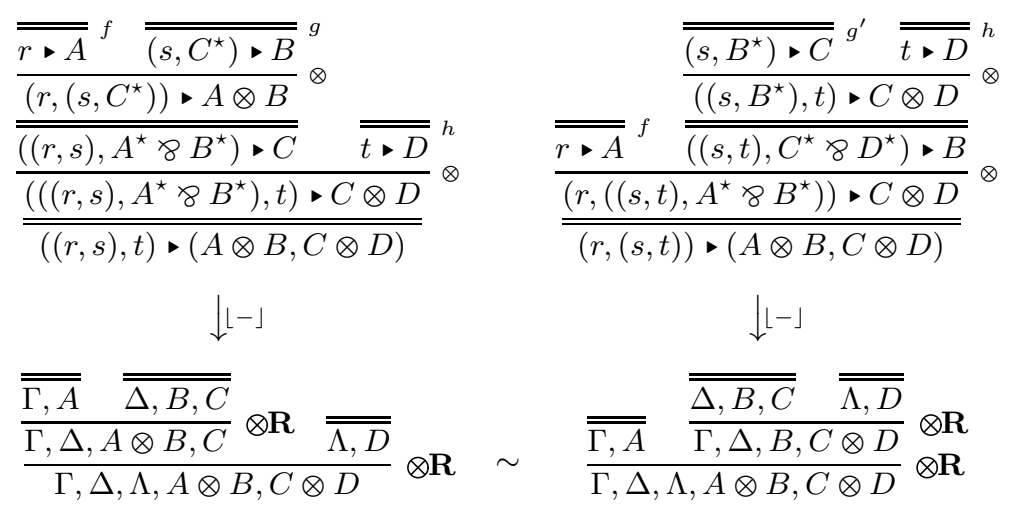

Fig. 14. A permutation in LT and LM

\section{Permutations}

A permutation in the sequent calculus LM is the exchange of two adjacent inferences. Proofs equal up to permutations, written $\Pi \sim \Pi^{\prime}$, construct the same proof net. For structured proofs, it must then be shown that parallel proofs $\pi$ and $\pi^{\prime}$ construct the same morphism whenever $\lfloor\pi\rfloor \sim\left\lfloor\pi^{\prime}\right\rfloor$. A permutation on LT-proofs will be one on the corresponding LM-proofs, as illustrated in Figure 14. In such permutations, the logical inferences that are permuted may be separated by equivariance derivations.

In working with permutations, since there are many inference rules in LT, there are 


\section{Cut-cut permutations}

$$
\begin{array}{ccc}
\frac{r ? \bullet(A, B) \quad s \bullet A^{\star} \quad t \bullet B^{\star}}{p ? \bullet q} & \frac{r ? \bullet(A, C) \quad s ? \bullet B \quad t ? \bullet D}{q ? \bullet(A \otimes B, C \otimes D)} \\
\frac{r ? \bullet A \quad s ? \bullet\left(A^{\star}, B\right) \quad t \bullet B^{\star}}{p ? \bullet q} & \frac{r ? \bullet A \quad s ? \bullet(B, C) \quad t ? \bullet D}{q ? \bullet(A \otimes B, C \otimes D)} \\
\frac{r ? \bullet A \quad s ? \bullet B \quad t ? \bullet\left(A^{\star}, B^{\star}\right)}{p ? \bullet q} & \frac{r ? \bullet A \quad s ? \bullet C \quad t ? \bullet(B, D)}{q ? \bullet(A \otimes B, C \otimes D)}
\end{array}
$$

Tensor-cut permutations

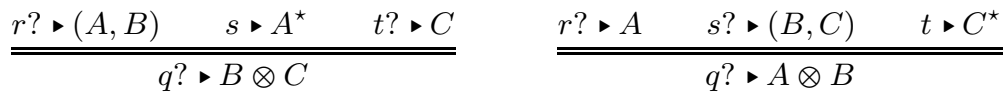

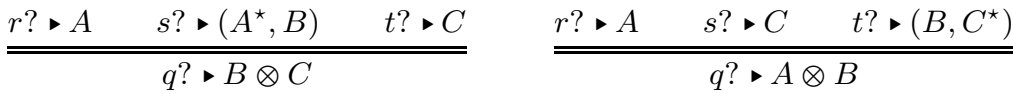

Fig. 15. Main permutation cases

potentially very many cases to verify. Narrowing these down, a first observation is that the $=$-inference rules in LT, corresponding to the $8 \mathbf{R}$-rule in LM, leave the map constructed by a proof unchanged; permutations involving these rules then trivially preserve this morphism. The focus is then on the binary LM-rules $\otimes \mathbf{R}$ and Cut. Permutation cases will be listed as abbreviated derivations from three premisses, one of which contains an active formula from both inferences. The example below corresponds to the permutation in Figure 14. What will be proved is that the possible ways of making the derivation concrete, are equivalent.

$$
\frac{r \bullet A \quad s \bullet(B, C) \quad t \bullet D}{(r,(s, t)) \bullet(A \otimes B, C \otimes D)}
$$

The main cases are listed in Figure 15, in three categories: tensor-tensor permutations, cut-cut permutations, and tensor-cut permutations. Within each category there are several cases, where the premiss containing two active formulae combines either the two left active formulae of both inferences, or one left and one right active formula, or both right active formulae. This generates all possible cases; however, there will be several sub-cases for each of the main cases (7)-(13), since for most premisses the antecedent may be empty or not - the exception being the right premiss of a composition ( $\mathbb{I}$ or $\circ$ ), which must contain more than one formula. The reason for differentiating cases in this way is that a permutation may, for example, change a tensor-inference into a virtual tensor-inference, as in case $(11 b)$, preventing the use of a simple list of permutations of 


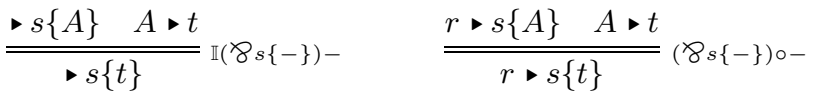

$$
\begin{aligned}
& \frac{\bullet t\{A\} \quad B}{-t\{A \otimes B\}} \mathbb{I}\left(8_{t\{A \otimes-\}) \quad} \frac{s \bullet t\{A\} \quad B}{s \triangleright t\{A \otimes B\}}-\circ(8 t\{A \otimes-\})\right. \\
& \frac{\bullet \quad(A, s) \bullet t}{s \bullet t}-\circ(-\otimes i d) \quad \frac{r \bullet(t, A) \quad s \bullet B}{(r, s) \bullet(t, A \otimes B)} s w \circ(-\otimes-)
\end{aligned}
$$

Fig. 16. Shorthand derivations

LT-inferences. Note that in Figure 15 the tree-sequents $q$ ? and $p ? \vee q$ are understood to contain the same annotated formulae as $r$ ?, $s$ ?, and $t$ ? combined.

\section{Abbreviations}

To simplify the presentation of permutation cases, several common derivations will be written in shortened form, as displayed in Figure 16. Tree-contexts $t\{-\}$ are used to generalise sequents such as $A \bullet B$ and $A \bullet(s, B)$ to $A \bullet t\{B\}$. That the abbrivations (14)-(17) construct the correct maps is a straightforward induction on $s\{-\}$ or $t\{-\}$; for example, the following two proofs construct the same morphism $\Phi\left(g^{\prime} \circ \Phi^{-1}(f)\right)=\left(i d \ngtr g^{\prime}\right) \circ f$, where $g^{\prime}=8 s\{g\}$.

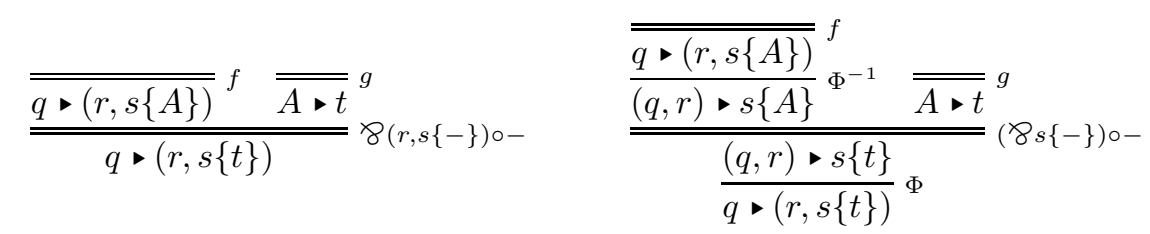

The derivation (18) constructs the correct morphism by the definition of the virtual tensor. Finally, the derivation (19) uses the switch natural transformation ( $s w)$, also known as linear distributivity (Cockett and Seely, 1997) or dissociativity (Došen and Petrić, 2005), given by the below diagram - this makes the abbreviation (19) correct by definition.

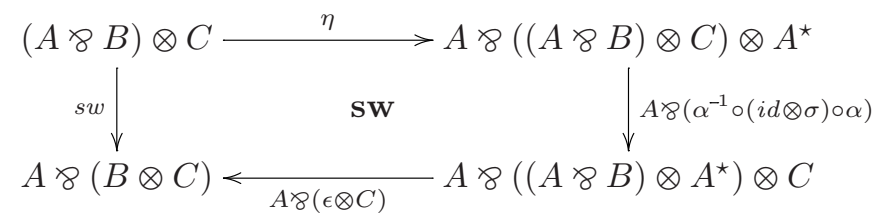

The transpose of the switch map, $\Phi(s w)$, is a map $i d \ngtr \eta$ (up to associativity and

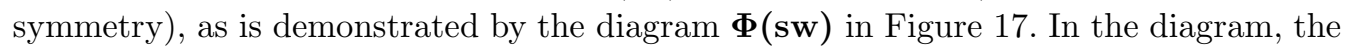
path along the top right is $\Phi(s w)$, and that along the bottom left is $i d \ngtr \eta$ followed by a coherence isomorphism for 8 . The big triangle in the diagram commutes by tracing the diagram $\mathbf{\Phi} \boldsymbol{\alpha} \mathbf{2}$ in Figure 13; the unnamed regions commute by naturality and $\eta \epsilon-$ cancellation. 


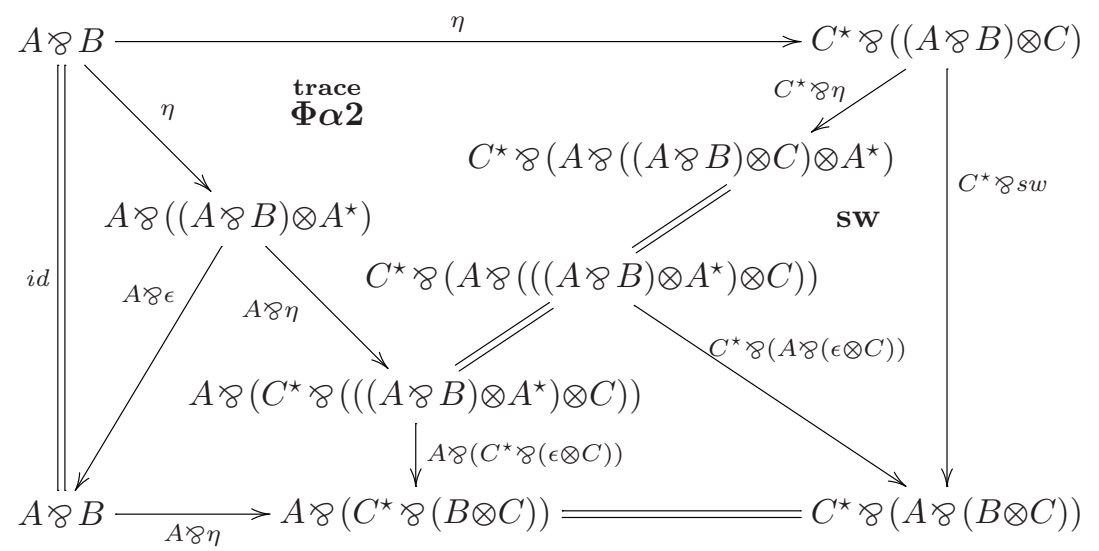

Fig. 17. The transpose of the switch map

$$
\frac{A^{\star} \bullet B \quad A \bullet s \quad B \bullet t}{s^{\star} \bullet t} \quad \frac{r \bullet(A, B) \quad A \bullet s \quad B \bullet t}{r \bullet(s, t)}
$$

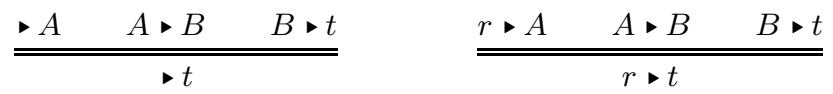

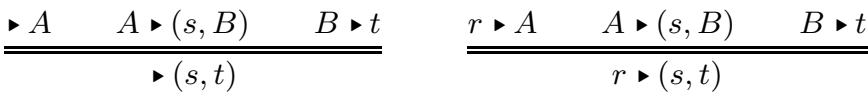

$$
\begin{aligned}
& \stackrel{\bullet A \quad \bullet B \quad(A, B) \bullet t}{\bullet t}
\end{aligned}
$$

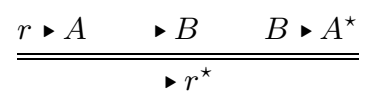

$\frac{r \bullet A \quad \bullet B \quad(A, B) \bullet t}{r \bullet t}$

$$
\frac{r \bullet A \quad s \bullet B \quad B \bullet A^{\star}}{s \bullet r^{\star}} \quad \frac{r \bullet A \quad s \bullet B \quad(A, B) \bullet t}{(r, s) \bullet t}
$$

Fig. 18. Cut-cut permutation cases

\section{Cut-cut permutations}

Lemma 8.1. The permutation of two cuts in an LT-proof preserves its denotation.

Proof. For each of the subcases of equations (4)-(6) in Figure 18, it must be shown that different instantiations of the derivation are equivalent. (The missing case in Figure 18 is that with premisses $\bullet A, \bullet$, and $B \bullet A^{\star}$, whose conclusion would be empty.) By duality of composition,

$$
\frac{s \bullet A \quad A \bullet t}{s \bullet t} \circ \quad \frac{t^{\star} \bullet A^{\star} \quad A^{\star} \bullet s^{\star}}{t^{\star} \bullet s^{\star}} \circ
$$




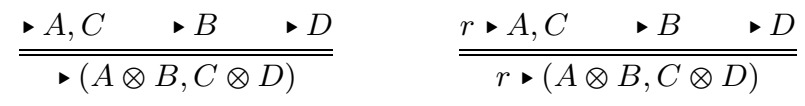

$$
\begin{aligned}
& \frac{\bullet A, C \quad \bullet B \quad t \bullet D}{t \bullet(A \otimes B, C \otimes D)} \quad \frac{r \bullet A, C \quad \bullet B \quad t \bullet D}{(r, t) \bullet(A \otimes B, C \otimes D)}
\end{aligned}
$$

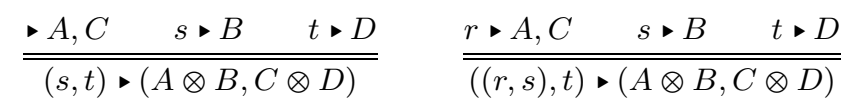

Fig. 19. Tensor-tensor permutation cases

the cases (4a) and (6d) are dual to (5b), cases (4b) and (6e) are dual to (5d), case (6b) is dual to (5a), and case (6c) is dual to (5c). This leaves five cases to be treated explicitly. Note that of these, only $5 \mathrm{~b}$ and $5 \mathrm{~d}$ apply to tensor-dual categories.

5a, 5c The following proofs construct the same map by functoriality of $\mathbb{I}$.

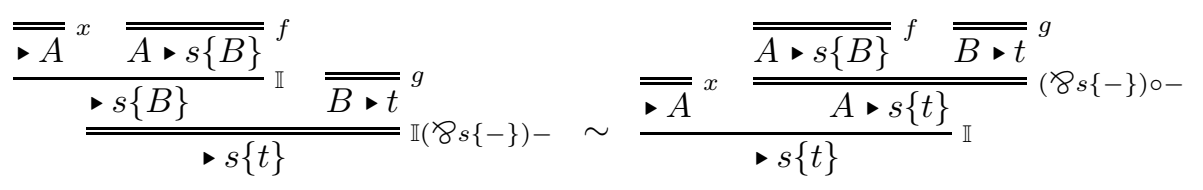

$\mathbf{5 b}, \mathbf{5 d}$ The following proofs have the same denotation by associativity of composition.

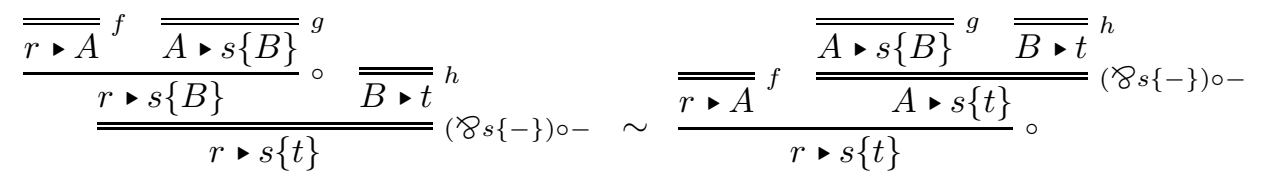

6a The following proofs have respective denotations $\mathbb{I}(g \circ \sigma)(y \otimes x)$ and $\mathbb{I} g(x \otimes y)$, which are equal by the diagram $\circledast \boldsymbol{\sigma}$ (the symmetry of $\odot)$.

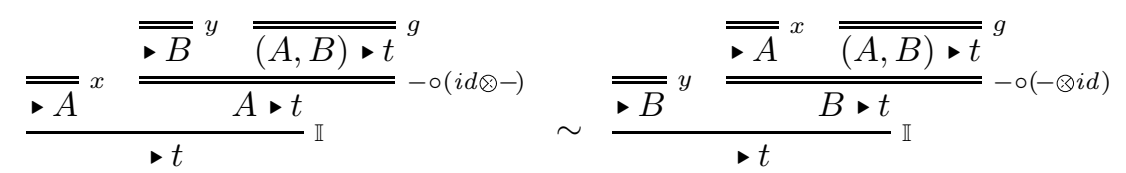

Tensor-tensor permutations

Lemma 8.2. The permutation of two tensor-inferences in an LT-proof preserves its denotation.

Proof. Figure 19 lists the subcases for derivation (7). The two other main cases for tensor-tensor permutations, (8) and (9), follow by symmetry of the tensor. Note that only (7c) and (7f) apply to tensor-dual categories. 
7a Both proofs below construct $\mathbb{I}((A \otimes y) \&(C \otimes z))(x)$.

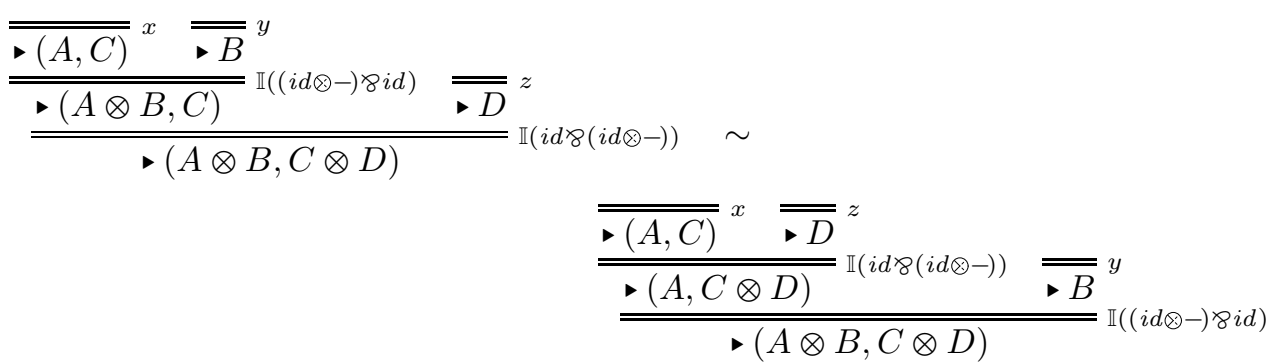

7b The maps constructed by the two following proofs are equal by the diagram below. The left region commutes by naturality of $\eta$; the equation that makes the right region commute, $(A \otimes y) \circ \lambda(x)=\lambda(\mathbb{I}(A \otimes y \diamond C)(x))$, is an instance of naturality of $\lambda$. The first proof corresponds to the path along the top of the diagram, the second to the path along the bottom.
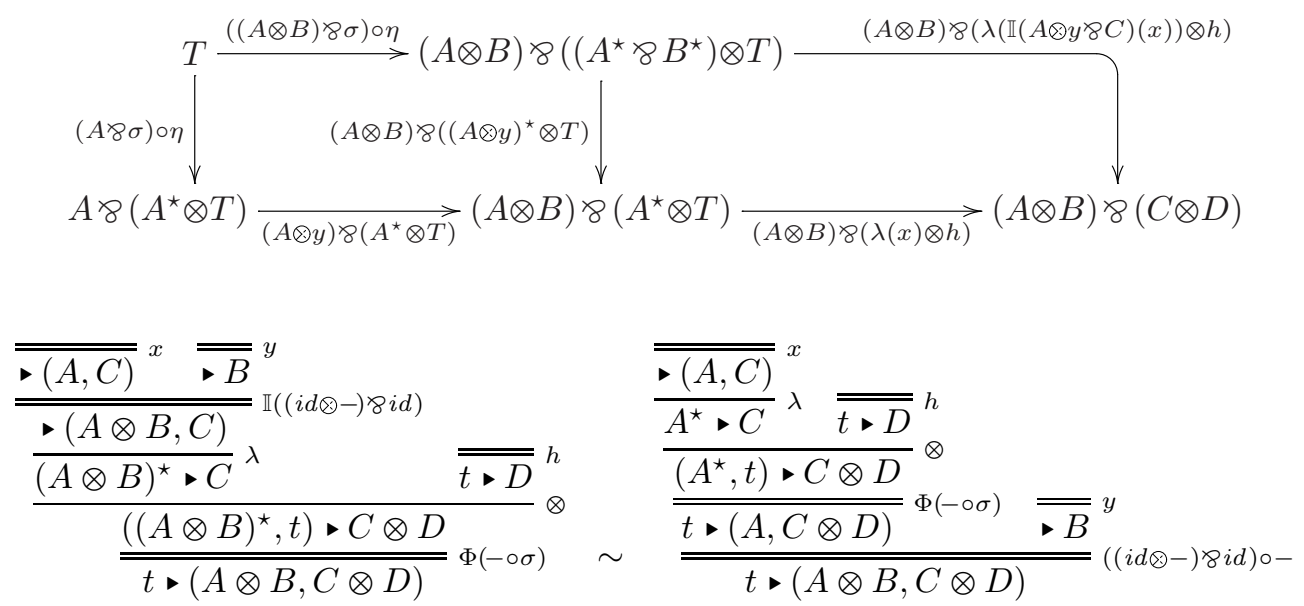

7c The two proofs below construct the following maps, denoted $\pi$ and $\rho$, where the identities on $A^{\star}, B$ and $D$ are taken for the maps $f, g$, and $h$ respectively.

$$
\begin{aligned}
\pi & =s w \circ\left(\left(\sigma^{\star} \circ \Phi((A \otimes B) \circ \sigma)\right) \otimes D\right) \\
& =s w \circ\left(\left(\sigma^{\star} \circ\left(A^{\star} \otimes \sigma\right) \circ \eta_{A}\right) \otimes D\right) \\
\rho & =\sigma^{\star} \circ s w \circ\left(\left(\sigma^{\star} \circ \Phi\left(\left(A^{\star} \otimes D\right) \circ \sigma\right)\right) \otimes B\right) \circ \sigma \\
& =\sigma^{\star} \circ s w \circ\left(\left(\sigma^{\star} \circ(A \ngtr \sigma)\right) \otimes B\right) \circ \sigma \circ(B \otimes \eta)
\end{aligned}
$$

The main diagram in Figure 20 shows the equation $\Phi(\pi)=\Phi(\rho)$, from which $\pi=\rho$ is immediate; equivalence for the general case (where $f, g$, and $h$ need not be identities) follows by naturality. The path along the top of the main diagram denotes $\Phi(\pi)$, while that along the left, bottom, and right denotes $\Phi(\rho)$. The region (1) commutes by naturality of $\eta_{A, B}: B \rightarrow A^{\star} \ngtr B \otimes A$ in $A$, i.e. the equation

$$
\left(f^{\star} \diamond B \otimes A\right) \circ \eta=(C \ngtr B \otimes f) \circ \eta
$$




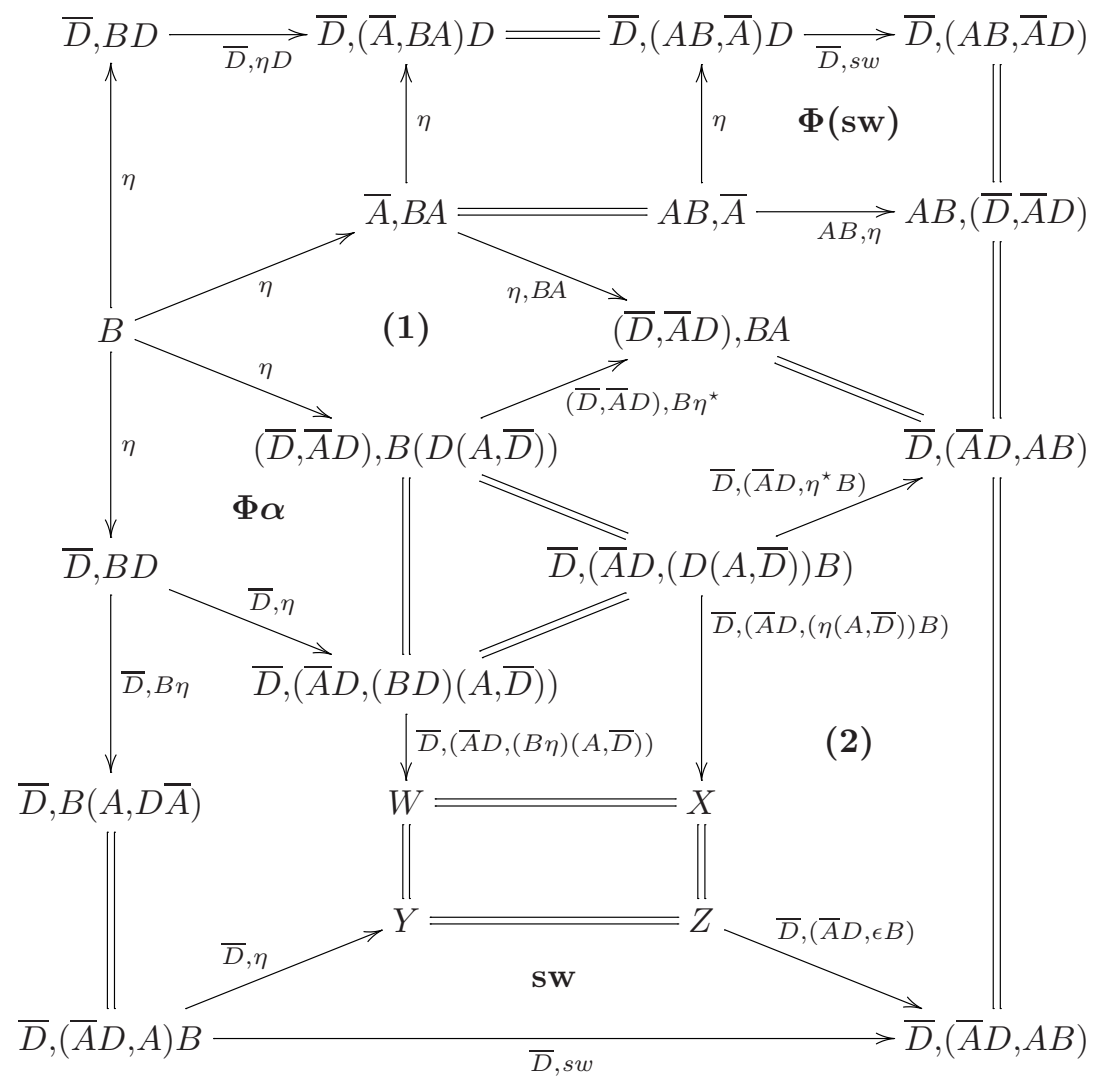

$$
\begin{aligned}
W & =\bar{D},(\bar{A} D,(B(A, D \bar{A}))(A, \bar{D})) \\
X & =\bar{D},(\bar{A} D,((A, D \bar{A})(A, \bar{D})) B) \\
Y & =\bar{D},(\bar{A} D,((\bar{A} D, A) B)(A, \bar{D})) \\
Z & =\bar{D},(\bar{A} D,((\bar{A} D, A)(A, \bar{D})) B)
\end{aligned}
$$

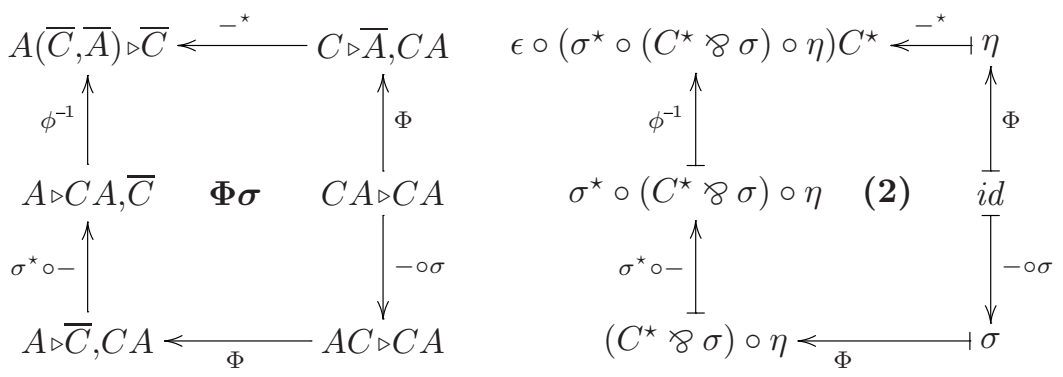

Fig. 20. Diagrams for permutation case (7c) 
(where $f: C \rightarrow A$ ) applied to $f=\eta^{\star}$. The region (2) commutes by tracing the diagram $\boldsymbol{\Phi} \boldsymbol{\sigma}$, as illustrated below the main diagram.

$$
\begin{aligned}
& {\overline{\overline{A^{\star} \cdot C}}}^{f}{ }^{\star}{ }^{\star} \quad \overline{\overline{s \bullet B}} g \\
& \frac{\left(C^{\star}, s\right) \bullet A \otimes B}{} \otimes
\end{aligned}
$$

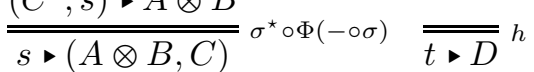

$$
\begin{aligned}
& (s, t) \bullet(A \otimes B, C \otimes D)
\end{aligned}
$$

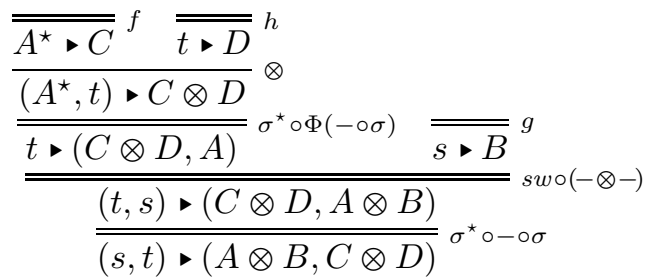

7d Both proofs below construct the map $(A \otimes y \ngtr B \otimes z) \circ f$.

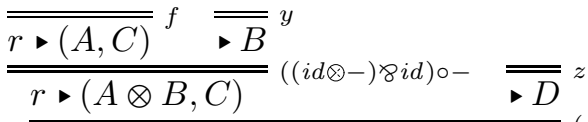

$$
\begin{aligned}
& r \vee(A \otimes B, C \otimes D) \quad(i d 8(i d \otimes-)) \circ-\quad \sim
\end{aligned}
$$

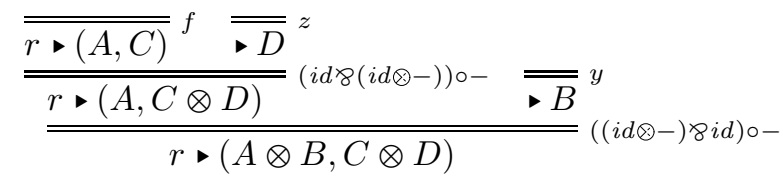

7e The maps constructed by the proofs below are equal by naturality of the switch map.

$$
\begin{aligned}
& \frac{\overline{\overline{r \bullet(A, C)}} f \quad \overline{\overline{\bullet B}}}{r \cdot(A \otimes B, C)}((i d \otimes-) \text { \&id }) \circ-\overline{\overline{t \bullet D}} h \\
& (r, t) \bullet(A \otimes B, C \otimes D) \quad s w \circ(-\otimes-) \quad \sim
\end{aligned}
$$

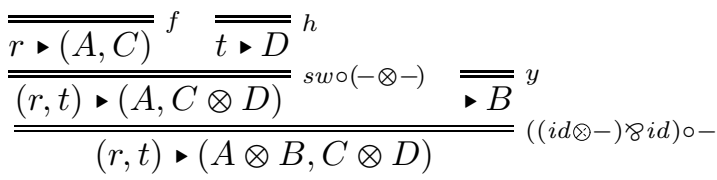

7f Equivalence of the proofs below is shown for the special case where $f=i d_{A 8 C}$, $g=i d_{B}$, and $h=i d_{D}$; the general case follows by naturality. The diagram in Figure 21 displays the transpose of the first proof along the top and right edges. The diagram shows that, up to coherence for the par ( 8 ), this map is equal to the map $\eta \ngtr \eta$, along the left edge. By a similar argument also the map constructed by the second proof is the 


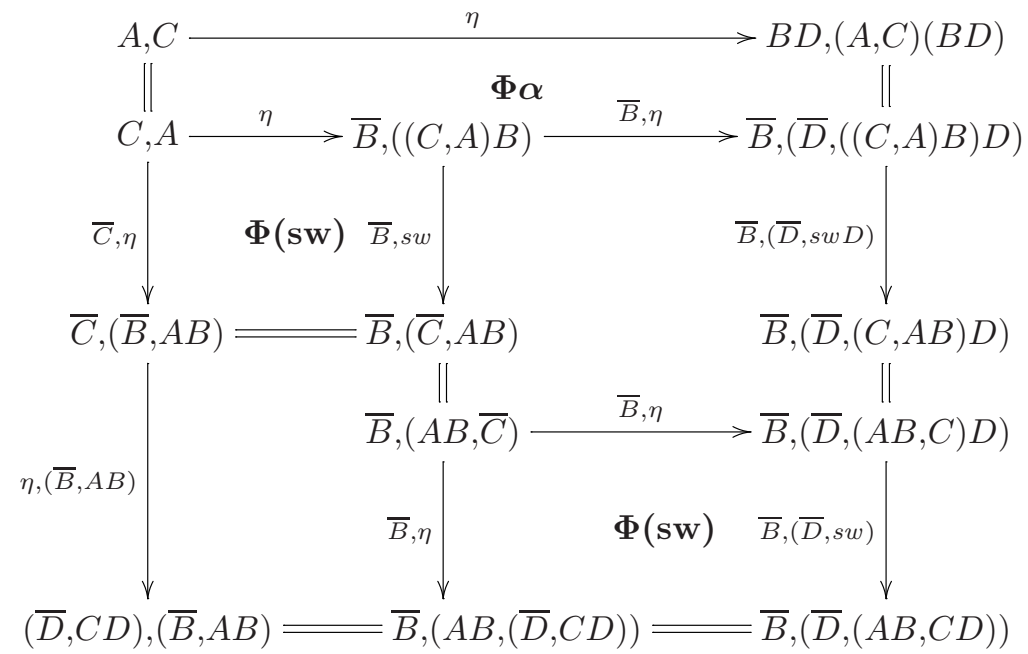

Fig. 21. The diagram for permutation case (7f)

transpose of $\eta \ngtr \eta$ (up to 8 -coherence).

$$
\begin{aligned}
& \overline{\overline{r \bullet(A, C)}} f \\
& \frac{(r, s) \bullet C, A \otimes B)}{(r w \circ(-\otimes-)} \\
& \frac{(r, s) \bullet C, \overline{t \bullet D}}{(r, s) \bullet(A \otimes B, C)} \sigma^{\star} \circ-\quad \\
& ((r, s), t) \bullet(A \otimes B, C \otimes D)
\end{aligned}
$$

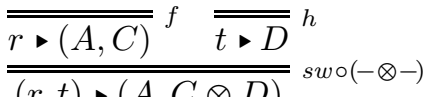

$$
\begin{aligned}
& \underline{\underline{\overline{(r, t) \bullet(A, C \otimes D)}}} \text { swo } \sigma^{\star} \circ- \\
& \overline{\overline{(r, t) \bullet(C \otimes D, A)}} \sigma^{\star} \circ-\quad \overline{\overline{s \triangleright B}^{g}} g \\
& \frac{((r, t), s) \bullet(C \otimes D, A \otimes B)}{\overline{((r, t), s) \bullet(A \otimes B, C \otimes D)}} \sigma^{\star} \circ-
\end{aligned}
$$

\section{Tensor-cut permutations}

Lemma 8.3. The permutation of a tensor-inference with a cut in an LT-proof preserves its denotation.

Proof. Figure 22 displays the subcases of permutations (10) and (11). The other two main cases, (12) and (13), follow from these two (respectively) by the symmetry of the tensor. By duality of composition, also (10a) follows from (11e), (10b) from (11f), (10c) from (11g), and (10d) from (11h). The subcases of (11) are treated below; for tensor-dual categories only the cases (11f) and (11h) apply. 


$$
\frac{A^{\star} \bullet B \quad A \bullet s \quad \bullet C}{s^{\star} \bullet B \otimes C} \quad \frac{A^{\star} \bullet B \quad A \bullet s \quad t \bullet C}{\left(s^{\star}, t\right) \bullet B \otimes C}
$$

$$
\frac{r \bullet(A, B) \quad A \bullet s \quad \bullet C}{r \bullet(s, B \otimes C)} \quad \frac{r \bullet(A, B) \quad A \bullet s \quad t \bullet C}{(r, t) \bullet(s, B \otimes C)}
$$

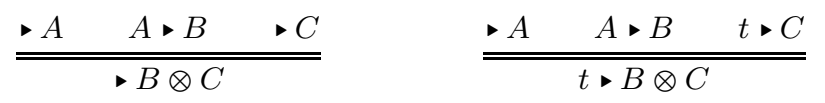

$$
\frac{\bullet \quad(A, s) \bullet B \quad \bullet C}{s \bullet B \otimes C} \quad \frac{\bullet A \quad(A, s) \bullet B \quad t \bullet C}{(s, t) \bullet B \otimes C}
$$

$$
\frac{r \bullet A \quad A \bullet B \quad \bullet C}{r \bullet B \otimes C} \quad \frac{r \bullet A \quad A \bullet B \quad t \bullet C}{(r, t) \bullet B \otimes C}
$$

$$
\frac{r \bullet A \quad(A, s) \bullet B \quad \bullet C}{(r, s) \bullet B \otimes C} \quad \frac{r \bullet A \quad(A, s) \bullet B \quad t \bullet C}{((r, s), t) \bullet B \otimes C}
$$

Fig. 22. Tensor-cut permutation cases

11a The following proofs are equivalent by the definition of (ब).

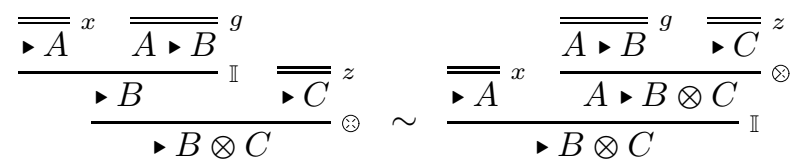

11b The proofs below construct $\mathbb{I} g(x) \otimes h$ and $(g \otimes h) \circ(x \otimes i d)$ respectively, which are equal by naturality for the virtual tensor.

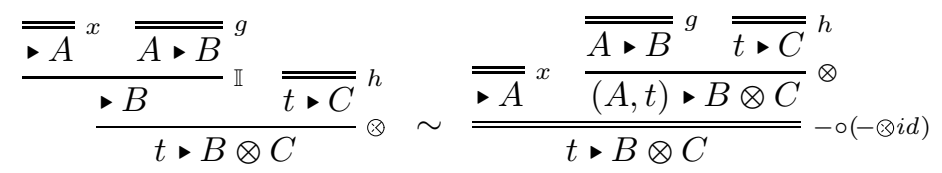

11c By naturality of the two virtual tensor operations, the maps constructed by both proofs are equal to $(i d \otimes z) \circ g \circ(x \otimes i d)$.

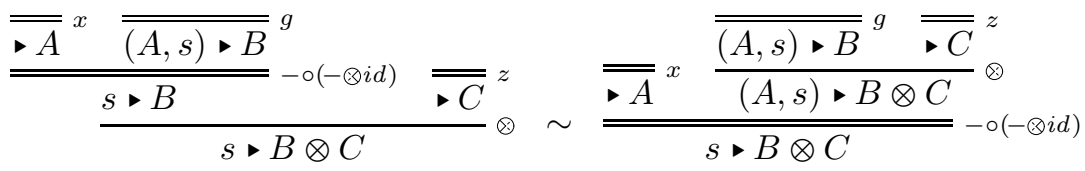

11d By naturality of $\Phi$ the map constructed by the second proof below, $\Phi^{-1}(\Phi(f \otimes$ $g) \circ(x \otimes S))$, is equal to $\Phi^{-1}(\Phi(f \otimes g)) \circ((x \otimes S) \otimes T)$, which after removing $\Phi^{-1} \circ \Phi$ is the map constructed by the first proof (note that $S$ and $T$ stand for the objects $\otimes s$ and 


$$
\begin{aligned}
& \stackrel{\bullet \quad \bullet B \quad(A, B) \bullet t}{\bullet t} \quad \frac{\bullet(A, B) \quad \bullet A \quad t \bullet B}{\bullet t}
\end{aligned}
$$

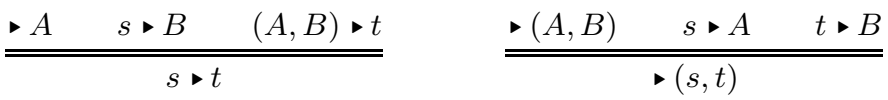

$$
\begin{aligned}
& \frac{r \bullet A \quad s \bullet B \quad(A, B) \bullet t}{(r, s) \bullet t} \quad \frac{r \bullet\left(A^{\star}, B^{\star}\right) \quad \bullet \quad t \bullet B}{r \bullet t^{\star}} \\
& \frac{r \bullet\left(A^{\star}, B^{\star}\right) \quad s \bullet A \quad t \bullet B}{r \bullet\left(s^{\star}, t^{\star}\right)}
\end{aligned}
$$

Fig. 23. Cut-elimination cases

$\otimes t)$

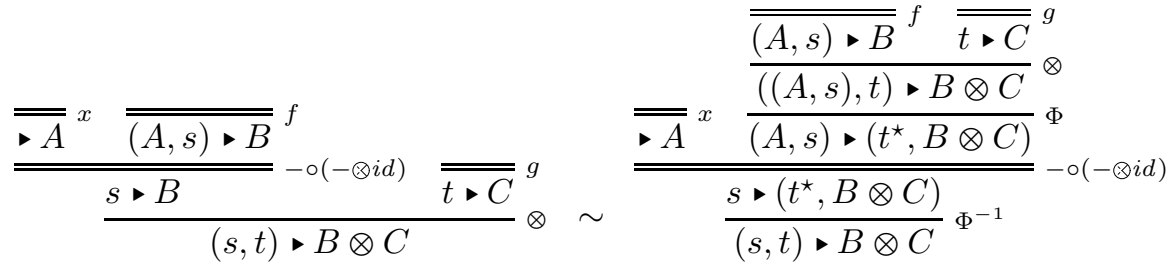

11e, 11g The following proofs are equivalent by the definition of (Q).

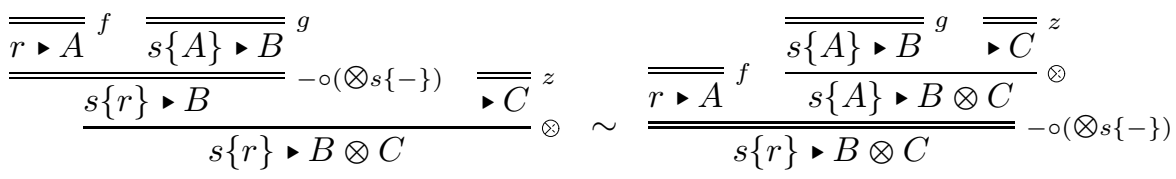

11f, 11h The following proofs are equivalent by functoriality of the tensor.

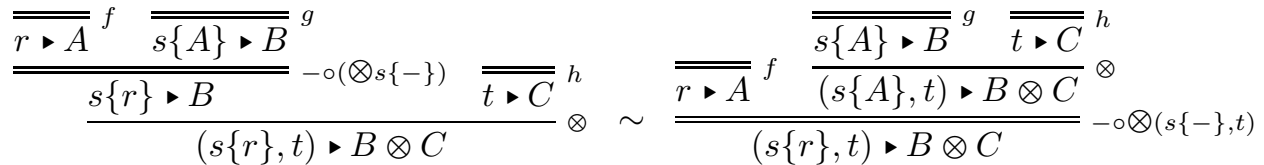

\section{Cut-elimination}

Cut elimination in LM, the calculus constructing proof nets, proceeds by the reduction steps in Figure 2, and preserves the net constructed by a proof. Here, it will be shown that the corresponding proof transformations in the structured calculus LT preserve the morphisms constructed by proofs.

Lemma 9.1. Cut-elimination steps preserve the denotation of LT-proofs.

Proof. Cuts in the calculus LT may be divided into three kinds, depending on whether the cut-formula is atomic, a tensor-formula, or a par-formula. For atomic cut formulae 
there are two cases, below, which are immediate.

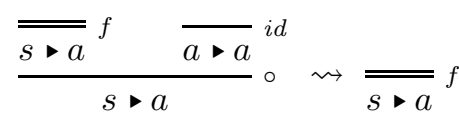

$$
\frac{\overline{a \triangleright a}^{i d} \overline{\overline{a \triangleright t}} g}{a \triangleright t} \circ \rightsquigarrow \overline{\overline{a \triangleright t}} g
$$

Figure 23 lists the cases for cuts on tensor-formula, (20a)-(20c), and for par-formulae, (21a)-(21d). Up to permutations there are two ways to instantiate each derivation: by two cuts, on the two active formulae $A$ and $B$; or by first combining the two derivations with one active formula by a tensor-inference. For each case these must be shown to be equivariant. The case (21d) is immediate from (20c) by duality; note that for tensor-dual categories only these two cases apply.

20a The following proofs are equivalent by the definition of (@).

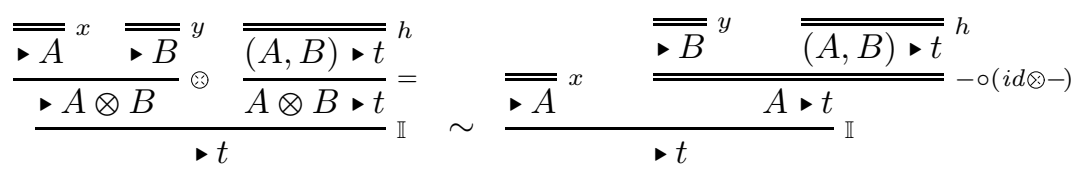

20b The following proofs are equivalent by naturality of (®)).

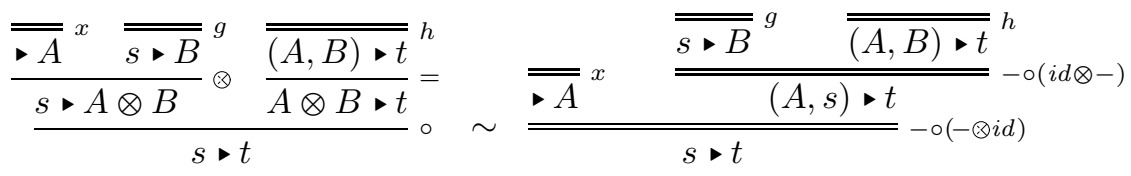

20c The following proofs are equivalent by functoriality of the tensor.

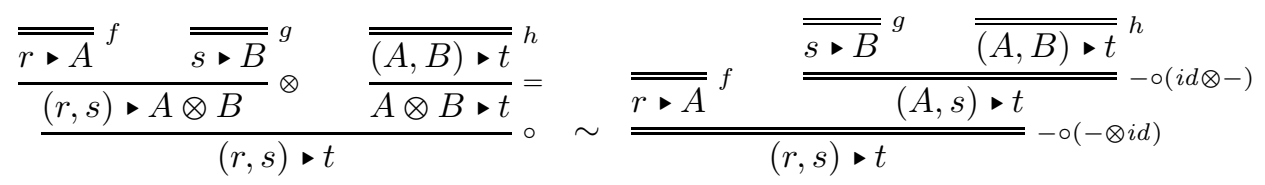

21a The main diagram in Figure 24 demonstrates that the map constructed by the first proof below, corresponding to the path along the top right of the diagram, is equal to the following map, along the left and bottom.

$$
\mathbb{I}\left(h^{\star} \circ \epsilon \circ \sigma\right)(y \otimes x)
$$

The unnamed regions in the diagram commute by naturality, functoriality, and by the definition of the virtual tensor. The region (1) is justified by tracing the diagram $\boldsymbol{\Phi} \boldsymbol{\sigma}$ for the identity on $A \otimes B$, as is done in the two diagrams below the main diagram. The above morphism is shown equal to that constructed by the second proof below, $\mathbb{I}\left(h^{\star} \circ \lambda(x)\right)(y)$, by the final diagram in Figure 24.

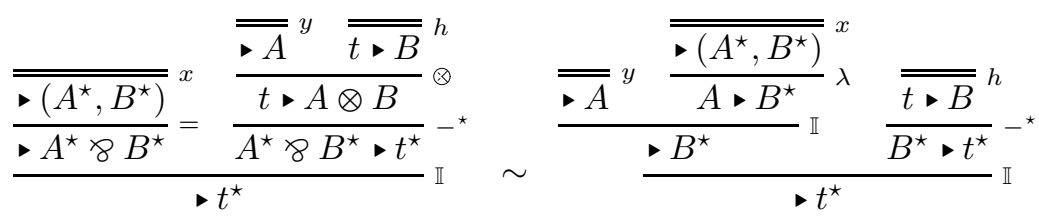



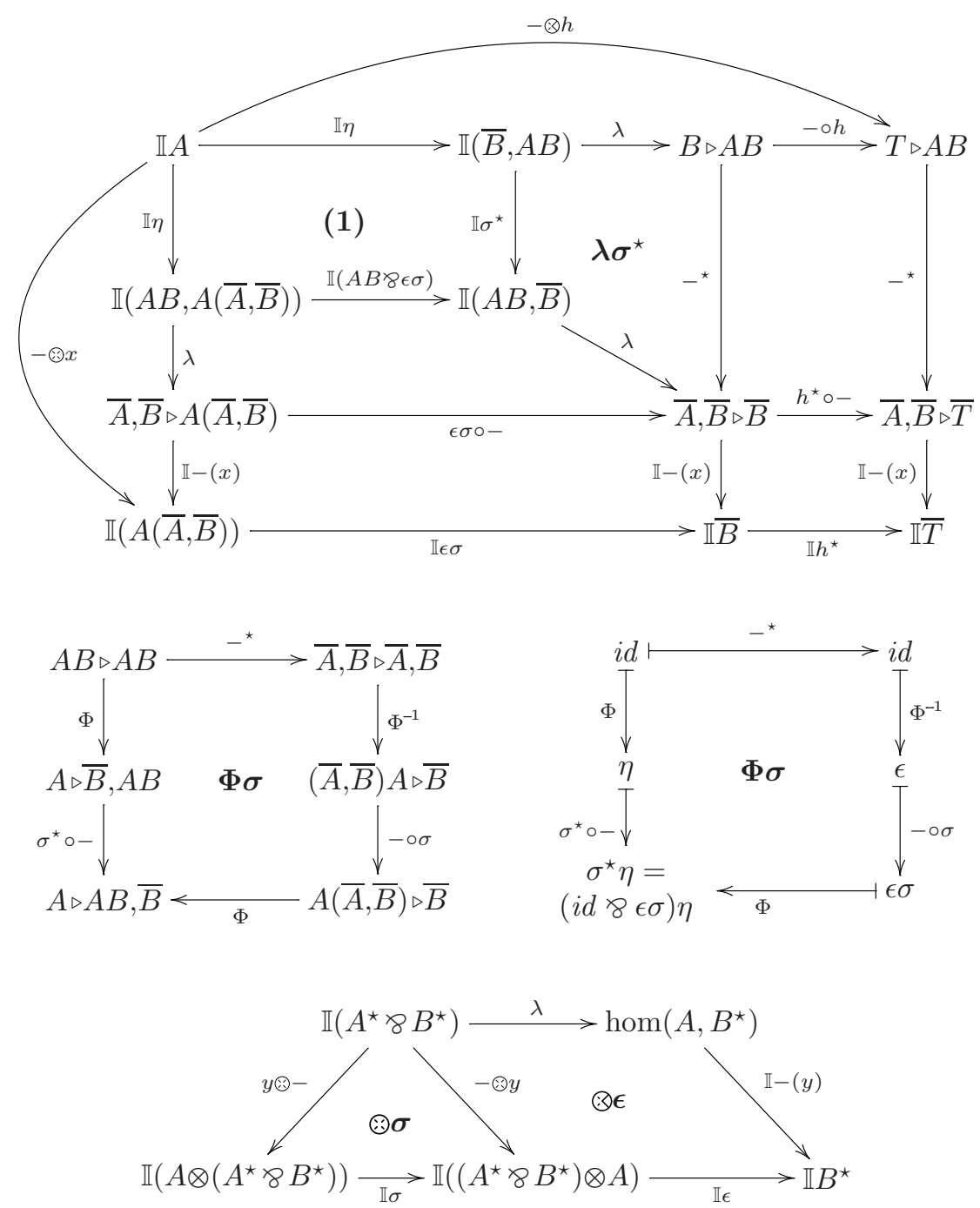

Fig. 24. Diagrams for elimination case (21a)

21b The following proofs are equivalent by functoriality of (४).

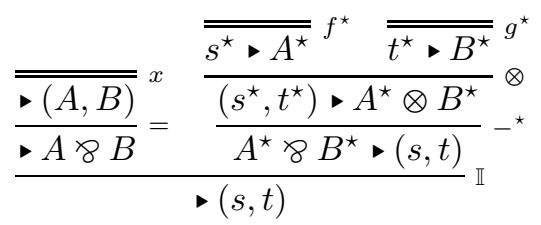

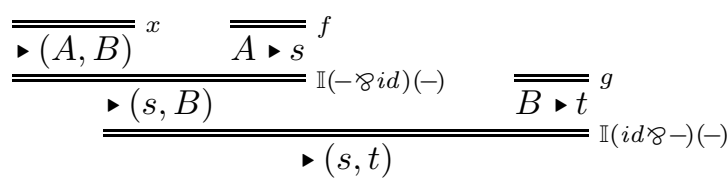


21c The case is dual to (20b): the map constructed by the first proof below is equal to the dual of $f^{\star} \circ(y \otimes h)$, which is equal to the map constructed by the second proof by naturality of the virtual tensor $(\otimes)$.

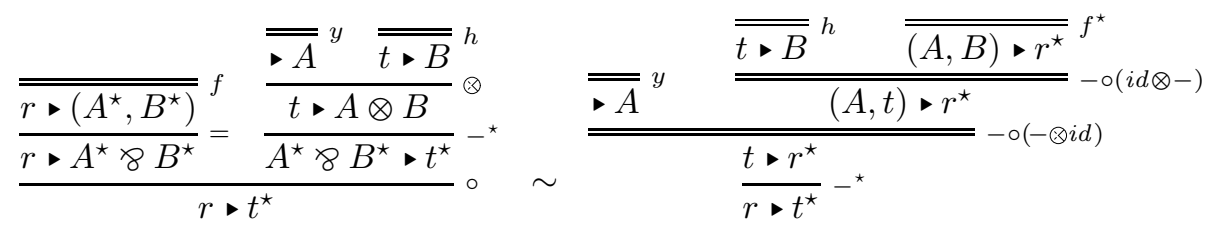

To conclude, it follows from the previous lemmata that proof nets factor out exactly permutations and cut-elimination in LT. The following proposition shows that $G_{1}$ and $\gamma$, in the proof of Theorem 5.10, are representable by functions from proof nets to morphisms, respectively vitual morphisms, in an SSA-category.

Proposition 9.2. If two LT-proofs $\pi$ and $\pi^{\prime}$ construct the same proof net, i.e., $\llbracket\lfloor\pi\rfloor \rrbracket=$ $\llbracket\left\lfloor\pi^{\prime}\right\rfloor \rrbracket$ (for a sequent $\vdash A$ or $A \vdash B$ ), then they construct the same SSA-morphism, i.e., $(\pi)_{F}=\left(\pi^{\prime}\right\rangle_{F}$ for any functor $F: \mathcal{A} \rightarrow \mathcal{C}$ into a ( $\partial$-strict) ssA-category $\mathcal{C}$.

Proof. It is standard (see e.g. (Lafont, 1995)) that two LM-proofs construct the same proof net if and only if they are equal up to permutations and cut-elimination. Correspondingly, two parallel LT-proofs $\pi$ and $\pi^{\prime}$ construct the same proof net if and only if there is a sequence $\pi=\pi_{1}, \pi_{2}, \ldots, \pi_{n}=\pi^{\prime}$ such that for each $\pi_{i}, \pi_{i+1}$ either $\left\lfloor\pi_{i}\right\rfloor=\left\lfloor\pi_{i+1}\right\rfloor$ or $\pi_{i}$ and $\pi_{i+1}$ are equal up to a permutation. In that case, $\pi$ and $\pi^{\prime}$ are equivalent by Proposition 7.3 and Lemmata 8.1, 8.2, 8.3, and 9.1. It follows that $\llbracket\lfloor\pi\rfloor \rrbracket=\llbracket\left\lfloor\pi^{\prime}\right\rfloor \rrbracket$ implies $(\pi)_{F}=\left(\pi^{\prime}\right)_{F}$.

\section{Acknowledgements}

We thank the anonymous referee for insightful, constructive remarks on this paper. Also, we cannot thank Robin Houston enough, for his careful explanation of the relationship between the virtual tensor and the promonoidal unit, and for contributing the proof of Proposition 5.6.

\section{References}

Barr, M. (1991). *-Autonomous categories and linear logic. Mathematical Structures in Computer Science, 1:159-178.

Blute, R. (1993). Linear logic, coherence, and dinaturality. Theoretical Computer Science, 115:341.

Blute, R., Cockett, R., Seely, R., and Trimble, T. (1996). Natural deduction and coherence for weakly distributive categories. Journal of Pure and Applied Algebra, 113:229-296.

Cockett, R. and Seely, R. (1997). Weakly distributive categories. Journal of Pure and Applied Algebra, pages 133-173.

Danos, V. and Regnier, L. (1989). The structure of multiplicatives. Archive for Mathematical Logic, 28:181-203. 
Day, B. (1970). On closed categories of functors. In Reports of the Midwest Category Seminar IV, volume 137 of Lecture Notes in Mathematics, pages 1-38. Springer Berlin / Heidelberg. Došen, K. and Petrić, Z. (2005). Proof-Net Categories. Polimetrica, Milan.

Girard, J.-Y. (1987). Linear logic. Theoretical Computer Science, 50(1):1-102.

Heijltjes, W. (2011). Proof nets for additive linear logic with units. In Proc. Logic in Computer Science (LICS), pages 207-216.

Heijltjes, W. and Houston, R. (2014). No proof nets for MLL: Proof equivalence in MLL with units is PSPACE-complete. In Proc. Logic in Computer Science (LICS). To appear.

Houston, R. (2008). Modelling linear logic without units. PhD thesis, University of Manchester.

Hughes, D. J. (2012). Simple free star-autonomous categories and full coherence. Journal of Pure and Applied Algebra, 216(11):2386-2410.

Joyal, A. and Street, R. (1991). The geometry of tensor calculus, i. Advances in Mathematics, $88: 55-112$.

Kelly, G. M. (1964). On MacLane's conditions for coherence of natural associators, commutativites, etc. Journal of Algebra, 1:397-402.

Kelly, G. M. (1982). Basic Concepts of Enriched Category Theory, volume 64 of London Mathematical Society Lecture Notes Series. Cambridge University Press.

Kelly, G. M. and Mac Lane, S. (1971). Coherence in closed categories. Journal of Pure and Applied Algebra, 1:97-140.

Lafont, Y. (1995). From proof nets to interaction nets. In Advances in Linear Logic, volume 222 of London Mathematical Society Lecture Notes, pages 225-247. Cambridge University Press.

Lamarche, F. and Straßburger, L. (2005). Constructing free Boolean categories. Proc. Logic in Computer Science (LICS), pages 209-218.

Lamarche, F. and Straßburger, L. (2006). From proof nets to the free *-autonomous category. Logical Methods in Computer Science, 2(4:3):1-44.

Mac Lane, S. (1963). Natural associativity and commutativity. Rice University Studies, 49:2846.

Selinger, P. (2011). A survey of graphical languages for monoidal categories. Lecture Notes in Physics, 813:289-355. 